\title{
Multivariable Feedback Particle Filter *
}

\author{
Tao Yang ${ }^{\mathrm{a}}$, Richard S. Laugesen ${ }^{\mathrm{b}}$, Prashant G. Mehta ${ }^{\mathrm{b}}$, Sean P. Meyn ${ }^{\mathrm{c}}$ \\ ${ }^{\mathrm{a}}$ GE Global Research, Niskayuna, NY, USA \\ ${ }^{\mathrm{b}}$ University of Illinois, Urbana-Champaign, IL, USA \\ ${ }^{\mathrm{c}}$ University of Florida, Gainesville, FL, USA
}

\begin{abstract}
This paper presents the multivariable extension of the feedback particle filter (FPF) algorithm for the nonlinear filtering problem in continuous-time. The FPF is a control-oriented approach to particle filtering. The approach does not require importance sampling or resampling and offers significant variance improvements; in particular, the algorithm can be applied to systems that are not stable. This paper describes new representations and algorithms for the FPF in the general multivariable nonlinear non-Gaussian setting. Theory surrounding the FPF is improved: Exactness of the FPF is established in the general setting, as well as well-posedness of the associated boundary value problem to obtain the filter gain. A Galerkin finite-element algorithm is proposed for approximation of the gain. Its performance is illustrated in numerical experiments.
\end{abstract}

Key words: Nonlinear filtering; Particle filtering; Estimation theory.

\section{Introduction}

In a recent work, we introduced a new feedback controlbased formulation of the particle filter for the nonlinear filtering problem $[55,54,56]$. The resulting filter is referred to as the feedback particle filter. In our prior journal article [56], the filter was described for the scalar case, where the signal and observation processes are both real-valued. The aim of this paper is to generalize the scalar results of our earlier paper to the multivariable filtering problem:

$$
\begin{aligned}
\mathrm{d} X_{t} & =a\left(X_{t}\right) \mathrm{d} t+\sigma\left(X_{t}\right) \mathrm{d} B_{t}, \\
\mathrm{~d} Z_{t} & =h\left(X_{t}\right) \mathrm{d} t+\mathrm{d} W_{t},
\end{aligned}
$$

where $X_{t} \in \mathbb{R}^{d}$ is the state at time $t, Z_{t} \in \mathbb{R}^{m}$ is the observation vector, and $\left\{B_{t}\right\},\left\{W_{t}\right\}$ are two mutually independent Wiener processes taking values in $\mathbb{R}^{d}$ and $\mathbb{R}^{m}$. The mappings $a(\cdot)$ : $\mathbb{R}^{d} \rightarrow \mathbb{R}^{d}, h(\cdot): \mathbb{R}^{d} \rightarrow \mathbb{R}^{m}$ and $\sigma(\cdot): \mathbb{R}^{d} \rightarrow \mathbb{R}^{d \times d}$ are $C^{1}$ functions. The covariance matrix of the observation noise $\left\{W_{t}\right\}$ is assumed to be positive definite. The function $h$ is a column vector whose $j$-th coordinate is denoted as $h_{j}$ (i.e., $\left.h=\left(h_{1}, h_{2}, \ldots, h_{m}\right)^{T}\right)$. By scaling, we assume without loss of generality that the covariance matrices associated with

\footnotetext{
* The conference version of this paper appeared in [53].

Email addresses: t.yangege.com (Tao Yang), laugesen@illinois.edu (Richard S. Laugesen), mehtapgeillinois.edu (Prashant G. Mehta), meyn@ufl. edu (Sean P. Meyn).
}

$\left\{B_{t}\right\},\left\{W_{t}\right\}$ are identity matrices. Unless otherwise noted, the stochastic differential equations (SDEs) are expressed in Itô form.

The objective of filtering is to estimate the posterior distribution of $X_{t}$ given the time history of observations $\mathscr{Z}_{t}:=$ $\sigma\left(Z_{s}: 0 \leq s \leq t\right)$. The density of the posterior distribution is denoted by $p^{*}$, so that for any measurable set $A \subset \mathbb{R}^{d}$,

$$
\int_{x \in A} p^{*}(x, t) \mathrm{d} x=\mathrm{P}\left\{X_{t} \in A \mid \mathscr{Z}_{t}\right\} .
$$

The filter is infinite-dimensional since it defines the evolution, in the space of probability measures, of $\left\{p^{*}(\cdot, t): t \geq\right.$ $0\}$. If $a(\cdot), h(\cdot)$ are linear functions, the solution is given by the finite-dimensional Kalman-Bucy filter. The article [7] surveys numerical methods to approximate the nonlinear filter. One approach described in this survey is particle filtering.

The particle filter is a simulation-based algorithm to approximate the filtering task [14]. The key step is the construction of $N$ stochastic processes $\left\{X_{t}^{i}: 1 \leq i \leq N\right\}$ : The value $X_{t}^{i} \in \mathbb{R}^{d}$ is the state for the $i$-th particle at time $t$. For each time $t$, the empirical distribution formed by, the particle population is used to approximate the posterior distribution. Recall that this is defined for any measurable set $A \subset \mathbb{R}^{d}$ by,

$$
p^{(N)}(A, t)=\frac{1}{N} \sum_{i=1}^{N} 1\left\{X_{t}^{i} \in A\right\} .
$$


A common approach in particle filtering is called sequential importance sampling, where particles are generated according to their importance weight at every time step $[2,14]$.

In our earlier papers [55,54,56], an alternative feedback control-based approach to the construction of a particle filter was introduced. The resulting particle filter, referred to as the feedback particle filter (FPF), was described for the scalar filtering problem (where $d=m=1$ ). The main result of this paper is to present the FPF for the multivariable filtering problem (1a)-(1b). In the following, this algorithm is described followed by a statement of the original contributions of this paper and comparison to relevant literature.

The feedback particle filter is a controlled system. The dynamics of the $i$-th particle have the following gain feedback form,

$$
\mathrm{d} X_{t}^{i}=a\left(X_{t}^{i}\right) \mathrm{d} t+\sigma\left(X_{t}^{i}\right) \mathrm{d} B_{t}^{i}+\underbrace{\mathrm{K}\left(X_{t}^{i}, t\right) \mathrm{d} l_{t}^{i}+\Omega\left(X_{t}^{i}, t\right) \mathrm{d} t}_{\mathrm{d} U_{t}^{i}},
$$

where $\left\{B_{t}^{i}\right\}$ are mutually independent standard Wiener processes, $I_{t}^{i}$ is similar to the innovation process that appears in the nonlinear filter,

$$
\mathrm{d} I_{t}^{i}:=\mathrm{d} Z_{t}-\frac{1}{2}\left(h\left(X_{t}^{i}\right)+\hat{h}\right) \mathrm{d} t,
$$

where $\hat{h}:=\mathrm{E}\left[h\left(X_{t}^{i}\right) \mid \mathscr{Z}_{t}\right]$. In a numerical implementation, we approximate $\hat{h} \approx \frac{1}{N} \sum_{i=1}^{N} h\left(X_{t}^{i}\right)=: \hat{h}^{(N)}$.

The gain function $\mathrm{K}$ is obtained by solving a weighted Poisson equation: For $j=1,2, \ldots, m$, the function $\phi_{j}$ is a solution to the second-order boundary value problem (BVP),

BVP

$$
\nabla \cdot\left(p(x, t) \nabla \phi_{j}(x, t)\right)=-\left(h_{j}(x)-\hat{h}_{j}\right) p(x, t),
$$

$$
\int \phi_{j}(x, t) p(x, t) \mathrm{d} x=0 \quad \text { (normalization), }
$$

for all $x \in \mathbb{R}^{d}$ where $\nabla$ and $\nabla \cdot$ denote the gradient and the divergence operators, respectively, and $p$ denotes the conditional density of $X_{t}^{i}$ given $\mathscr{Z}_{t}$, and $\hat{h}_{j}:=\mathrm{E}\left[h_{j}\left(X_{t}^{i}\right) \mid \mathscr{Z}_{t}\right]$. Although this paper is limited to $\mathbb{R}^{d}$, for domains with boundary, the BVP is accompanied by a Neumann boundary condition,

$$
\nabla \phi(x, t) \cdot \hat{n}(x)=0,
$$

for all $x$ on the boundary of the domain where $\hat{n}(x)$ is a unit normal vector at the boundary point $x$.

In terms of BVP solution, the gain function is given by

$$
[\mathrm{K}]_{l j}=\frac{\partial \phi_{j}}{\partial x_{l}} .
$$

Note that the gain function $\mathrm{K}$ is matrix-valued (with dimension $d \times m$ ) and it needs to be obtained for each value of time $t$. Also recall that $h$ is a column vector with $m$ entries.
Finally, $\Omega=\left(\Omega_{1}, \Omega_{2}, \ldots, \Omega_{d}\right)^{T}$ is the Wong-Zakai correction term:

$$
\Omega_{l}(x, t):=\frac{1}{2} \sum_{k=1}^{d} \sum_{s=1}^{m} \mathrm{~K}_{k s}(x, t) \frac{\partial \mathrm{K}_{l s}}{\partial x_{k}}(x, t) .
$$

The controlled system (2)-(6) is called the multivariable feedback particle filter.

The inspiration for controlling a single particle - via the control input $U_{t}^{i}$ in (2) - comes from the mean-field game formalism; cf., $[25,57]$. With no control input $\left(U_{t}^{i}=0\right)$, the particle system (2) implements a Monte Carlo propagation of the (un-conditioned) probability distribution for (1a). One interpretation of the control input $U_{t}^{i}$ is that it implements the 'Bayesian update step' to account for conditioning due to observations (1b). The gain times error structure is reminiscent of the Bayesian update formula in the Kalman filter (See also Remark 1). Structurally, such an update procedure is very different from the importance sampling based implementation of the Bayes rule in conventional particle filters. While the FPF is naturally a continuous-time algorithm, an importance sampling-based procedure typically requires discretization of time; cf., [2]. In discrete-time, approximations of the posterior distribution are typically used as importance densities.

The contributions of this paper are as follows:

- Exactness. The feedback particle filter (2) is shown to be exact, given an exact initialization $p(\cdot, 0)=p^{*}(\cdot, 0)$. Consequently, if the initial conditions $\left\{X_{0}^{i}\right\}_{i=1}^{N}$ are drawn from the initial density $p^{*}(\cdot, 0)$ of $X_{0}$, then, as $N \rightarrow \infty$, the empirical distribution of the particle system approximates the posterior density $p^{*}(\cdot, t)$ for each $t$.

- Well-posedness. A weak formulation of the BVP (4) is introduced, and used to prove an existence-uniqueness result for $\phi_{j}$ in a suitable function space. Certain apriori bounds are derived for the gain function to show that the resulting control input in (2) is admissible (That is, the filter (2) is well-posed in the Itô sense).

- Numerical algorithms. Based on the weak formulation, a Galerkin finite-element algorithm is proposed for approximation of the gain function $\mathrm{K}(x, t)$. The algorithm is completely adapted to data (that is, it does not require an explicit approximation of $p(x, t)$ or computation of derivatives). Certain closed-form expressions for the gain function are derived in a few special cases. The conclusions are illustrated with numerical examples.

In recent years, there has been a burgeoning interest in application of ideas and techniques from statistical mechanics to nonlinear estimation and control theory. Although some of these applications are classical (see e.g., Del Moral $[37,11,13,12])$, the recent impetus comes from explosive interest in mean-field games, starting with the two 


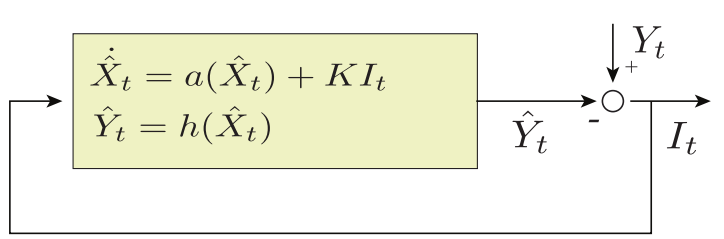

(a): Kalman Filter

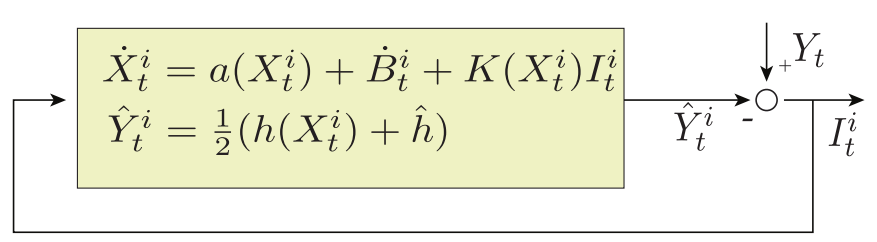

(b): Feedback Particle Filter

Fig. 1. Innovation error-based feedback structure for the (a) Kalman filter and (b) nonlinear feedback particle filter.

papers from 2007: Lasry and Lions paper titled "Mean-field games" [31] and a paper in IEEE TAC by Huang, Caines and Malhamé [25]. These papers spurred interest in analysis and synthesis of controlled interacting particle systems.

For the continuous-time filtering problem, an approximate particle filtering algorithm appears in the 2009 paper of Crisan and Xiong [8]. In the 2003 paper of Mitter, an optimal control problem for particle filtering is formulated based on duality [36]. A comparison between the algorithms proposed in these papers and the feedback particle filter appears in [56]. Certain mean-field game inspired approximate algorithms for nonlinear estimation appear in $[20,40,19]$. In discrete-time settings, Daum and Huang have introduced the particle flow filter algorithm [9]. A detailed comparison of the feedback particle filter to Daum's particle flow filter appears in [52]. There are by now a growing list of papers on application of such controlled algorithms to: physical activity recognition [50,49], estimation of soil parameters in dredging applications [45], estimation and control in the presence of communication channels [34], target state estimation $[9,48]$, satellite tracking [6] and weather forecasting [42].

The outline of the remainder of this paper is as follows. The nonlinear filter is introduced and shown to be exact in Sec. 2. The weak formulation of the BVP appears in Sec. 3 where well-posedness results are derived and the numerical Galerkin algorithm is described. A self-contained summary of the finite- $N$ FPF algorithm appears in Sec. 4 with the linear Gaussian example discussed as a special case. Two numerical examples appear in Sec. 5 followed by conclusions in Sec. 6.

Notation: $C^{k}$ is used to denote the space of $k$-times continuously differentiable functions. $L^{\infty}$ is used to denote the space of functions that are bounded a.e. (Lebesgue); $L^{2}\left(\mathbb{R}^{d} ; p\right)$ the Hilbert space of functions on $\mathbb{R}^{d}$ that are square-integrable with respect to density $p ; H^{k}\left(\mathbb{R}^{d} ; p\right)$ denotes the Hilbert space of functions whose first $k$ derivatives (defined in the weak or distributional sense [17]) are in $L^{2}\left(\mathbb{R}^{d} ; p\right)$, and $H_{0}^{1}\left(\mathbb{R}^{d} ; p\right) \doteq\left\{\phi \in H^{1}\left(\mathbb{R}^{d} ; p\right) \mid \int \phi(x) p(x) \mathrm{d} x=0\right\}$.

For a function $f, \nabla f=\frac{\partial f}{\partial x_{i}}$ is used to denote the gradient, and $D^{2} f=\frac{\partial^{2} f}{\partial x_{i} x_{j}}$ is used to denote the Hessian. For a vector $v, v^{T}$ denotes the transpose of $v$, and $\nabla \cdot v=\sum_{i=1}^{d} \frac{\partial v_{i}}{\partial x_{i}}$ is used to denote the divergence. The derivatives are interpreted in the weak sense.

\section{Multivariable Feedback Particle Filter}

Consider the continuous time filtering problem $(1 \mathrm{a}, 1 \mathrm{~b})$ introduced in Sec. 1. Denote as $p^{*}(x, t)$ the conditional density of $X_{t}$ given $\mathscr{Z}_{t}=\sigma\left(Z_{s}: 0 \leq s \leq t\right)$. The evolution of $p^{*}(x, t)$ is described by the Kushner-Stratonovich $(\mathrm{K}-\mathrm{S})$ equation $[30,46]$ :

$$
\mathrm{d} p^{*}=\mathscr{L}^{\dagger} p^{*} \mathrm{~d} t+(h-\hat{h})^{T}\left(\mathrm{~d} Z_{t}-\hat{h} \mathrm{~d} t\right) p^{*},
$$

where $\hat{h}=\int h(x) p^{*}(x, t) \mathrm{d} x$ and $\mathscr{L}^{\dagger} p^{*}=-\nabla \cdot\left(p^{*} a\right)+$ $\frac{1}{2} \sum_{l, k=1}^{d} \frac{\partial^{2}}{\partial x_{l} \partial x_{k}}\left(p^{*}\left[\sigma \sigma^{T}\right]_{l k}\right)$.

\subsection{A control architecture for particle filter}

The model for the particle filter is given by the Itô sde,

$$
\mathrm{d} X_{t}^{i}=a\left(X_{t}^{i}\right) \mathrm{d} t+\sigma\left(X_{t}^{i}\right) \mathrm{d} B_{t}^{i}+\underbrace{u\left(X_{t}^{i}, t\right) \mathrm{d} t+\mathrm{K}\left(X_{t}^{i}, t\right) \mathrm{d} Z_{t}}_{\mathrm{d} U_{t}^{i}},
$$

where $X_{t}^{i} \in \mathbb{R}^{d}$ is the state for the $i$-th particle at time $t$, and $\left\{B_{t}^{i}\right\}$ are mutually independent standard Wiener processes. The initial condition $X_{0}^{i}$ is drawn from the initial density $p^{*}(x, 0)$ of $X_{0}$ independent of $\left\{B_{t}^{i}\right\}$. Both $\left\{B_{t}^{i}\right\}$ and $\left\{X_{0}^{i}\right\}$ are also assumed to be independent of $X_{t}, Z_{t}$. Note that the gain function $\mathrm{K}(x, t)$ is a $d \times m$ matrix and $u(x, t) \in \mathbb{R}^{d}$.

Certain admissibility requirements are imposed on the control input $U_{t}^{i}$ in (8):

Definition 1 (Admissible Input) The control input $U_{t}^{i}$ is admissible if the random variables $u(x, t)$ and $\mathrm{K}(x, t)$ are $\mathscr{Z}_{t}$ measurable for each $t$. And for each $t, \mathrm{E}[|u|]:=$ $\mathrm{E}\left[\sum_{l}\left|u_{l}\left(X_{t}^{i}, t\right)\right|\right]<\infty$ and $\mathrm{E}\left[|\mathrm{K}|^{2}\right]:=\mathrm{E}\left[\sum_{l j}\left|\mathrm{~K}_{l j}\left(X_{t}^{i}, t\right)\right|^{2}\right]<$ $\infty$.

The conditional density of $X_{t}^{i}$ given the filtration $\mathscr{Z}_{t}$ is denoted as $p(x, t)$. Its evolution equation is described in the next result. The proof is identical to the proof in the scalar case (see Proposition 3.1 in [56]). It is omitted here.

Proposition 1 Consider the process $X_{t}^{i}$ that evolves according to the particle filter model (8). The conditional density 
of $X_{t}^{i}$ given the filtration $\mathscr{Z}_{t}$, denoted as $p(x, t)$, satisfies the forward equation:

$$
\begin{array}{r}
\mathrm{d} p=\mathscr{L}^{\dagger} p \mathrm{~d} t-\nabla \cdot(p \mathrm{~K}) \mathrm{d} Z_{t}-\nabla \cdot(p u) \mathrm{d} t \\
+\frac{1}{2} \sum_{l, k=1}^{d} \frac{\partial^{2}}{\partial x_{l} \partial x_{k}}\left(p\left[\mathrm{KK}^{T}\right]_{l k}\right) \mathrm{d} t .
\end{array}
$$

Problem Statement: Recall that there are two types of conditional densities of interest in our analysis:

(1) $p(x, t)$ : Defines the conditional density of $X_{t}^{i}$ given $\mathscr{Z}_{t}$.

(2) $p^{*}(x, t)$ : Defines the conditional density of $X_{t}$ given $\mathscr{Z}_{t}$.

The functions $\{u(x, t), \mathrm{K}(x, t)\}$ are said to be exact if $p \equiv p^{*}$. That is, given $p^{*}(\cdot, 0)=p(\cdot, 0)$, the goal is to choose $\{u, \mathrm{~K}\}$ in the particle filter model (8) so that the evolution equations of these conditional densities coincide (see (7) and (9)).

Solution: The feedback particle filter algorithm introduced in Sec. 1 represents the following choice of functions $\{u, \mathrm{~K}\}$ : The function $\mathrm{K}$ is a solution to the BVP (4)-(5) and the function $u$ is obtained as

$$
u(x, t)=-\frac{1}{2} \mathrm{~K}(x, t)(h(x)+\hat{h})+\Omega(x, t) .
$$

Substituting (10) into (8) gives the feedback particle filter model (2)-(3) in Sec. 1. The reader is referred to our earlier paper [56] for additional justification regarding these choices.

The above choice of $\{u, \mathrm{~K}\}$ leads to an exact filter under certain additional technical assumptions to ensure admissibility of the control input $U_{t}^{i}$. The statement of these assumptions together with the consistency result appear in the following section. The proof of admissibility appears in Sec. 3.

\subsection{Consistency with the nonlinear filter}

To establish admissibility of the input $U_{t}^{i}$ requires additional assumptions on the density $p$ and the function $h$ :

(i) Assumption $\boldsymbol{A 1}$ The function $h \in C^{1}$ with $h, \nabla h \in$ $L^{2}\left(\mathbb{R}^{d}, p\right)$.

(ii) Assumption $A 2$ The probability density $p(x, t)$ is of the form $p(x, t)=e^{-\mathscr{G}(x, t)}$ and admits a spectral gap (or Poincaré inequality) with constant $\lambda \geq c_{1}$ [26]: That is, for all functions $\phi \in H_{0}^{1}\left(\mathbb{R}^{d} ; p\right)$,

$$
\int|\phi(x)|^{2} p(x, t) \mathrm{d} x \leq \frac{1}{\lambda} \int|\nabla \phi(x)|^{2} p(x, t) \mathrm{d} x .
$$

(iii) Assumption A3 The second derivatives of $\mathscr{G}(x, t)$ with respect to $x$ are uniformly bounded, i.e., $D^{2} \mathscr{G} \in L^{\infty}$.
The following theorem then shows that the two evolution equations (7) and (9) are identical. The proof appears in Appendix A.1.

Theorem 1 Consider the two evolution equations for $p$ and $p^{*}$, defined according to the solution of the forward equation (9) and the $K-S$ equation (7), respectively. Suppose that Assumptions (A1)-(A3) hold and that the gain function $\mathrm{K}(x, t)$ is obtained according to (4)-(5). Then, provided $p(\cdot, 0)=p^{*}(\cdot, 0)$, we have for all $t \geq 0$,

$$
p(\cdot, t)=p^{*}(\cdot, t)
$$

\subsection{Some remarks}

Remark 1 Stratonovich form of the feedback particle filter. In the Stratonovich form, the filter admits a simpler representation,

$$
\mathrm{d} X_{t}^{i}=a\left(X_{t}^{i}\right) \mathrm{d} t+\sigma\left(X_{t}^{i}\right) \mathrm{d} B_{t}^{i}+\mathrm{K}\left(X^{i}, t\right) \circ\left(\mathrm{d} Z_{t}-\frac{1}{2}\left(h\left(X_{t}^{i}\right)+\hat{h}\right) \mathrm{d} t\right) .
$$

Given that the Stratonovich form provides a mathematical interpretation of the (formal) ODE model [39, Section 3.3

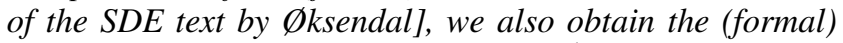
$O D E$ model of the filter. Denoting $Y_{t} \doteq \frac{\mathrm{d} Z_{t}}{\mathrm{~d} t}$ and white noise process $\dot{B}_{t}^{i} \doteq \frac{\mathrm{d} B_{t}^{i}}{\mathrm{~d} t}$, the ODE model of the filter is given by,

$$
\frac{\mathrm{d} X_{t}^{i}}{\mathrm{~d} t}=a\left(X_{t}^{i}\right)+\sigma\left(X_{t}^{i}\right) \dot{B}_{t}^{i}+\mathrm{K}\left(X^{i}, t\right) \cdot\left(Y_{t}-\frac{1}{2}\left(h\left(X_{t}^{i}\right)+\hat{h}\right)\right) .
$$

The feedback particle filter thus provides a generalization of the Kalman filter to nonlinear systems, where the innovation error-based feedback structure of the control is preserved (see Fig. 1). For the linear Gaussian case, it is shown in Sec. 4.2 that the gain function is the Kalman gain. For the nonlinear case, the Kalman gain is replaced by a nonlinear function of the state.

Remark 2 Remarks on Assumptions A1-A3. The spectral gap condition $A 2$ is important to show existence, uniqueness and regularity of the solutions of the BVP (see Appendix A.2). It is akin to the 'apriori energy bound' that one needs to establish well-posedness of the solutions of elliptic partial differential equations; $c f$. , [17].

For example, if the density $p$ is Gaussian with the covariance matrix $\Sigma$, the spectral gap constant $(1 / \lambda)$ equals the largest eigenvalue of $\Sigma$. For a general class of $p$, the Assumptions A2-A3 hold if $p$ has a Gaussian tail, i.e., $p$ behaves as a Gaussian for large $|x|$. This is because $\mathscr{G}$ is quadratic in $x$ and so its second derivatives are bounded, implying Assumption A3. The spectral gap Assumption A2 holds also, because $\nabla \mathscr{G}$ grows linearly for large $|x|$ and so $|\nabla \mathscr{G}|^{2}-$ $2 \Delta \mathscr{G} \rightarrow \infty$ as $|x| \rightarrow \infty$, which is known to imply existence of 
a spectral gap [24, §3.2]. The assumption A1 then holds for polynomial functions $h$.

For the nonlinear filtering problem, we conjecture that if assumptions A1-A3 hold for $p(x, 0)$ then they continue to hold for $p(x, t)$ for all $0<t<t_{f}$, with constants that are worse (that is, with a smaller $c_{1}$ value in $A 2$ and with a larger $L^{\infty}$ norm in A3). Such is the case for the linear Gaussian filtering problem. In [32], it is also shown to be true for the nonlinear non-Gaussian case with a constant signal model.

Remark 3 Convergence to the mean-field limit. Thm. 1 is based on the mean-field setting in which the gain $\mathrm{K}\left(X_{t}^{i}, t\right)$ is obtained as a function of the posterior $p=p^{*}$ for $X_{t}^{i}$, and $\hat{h}$ is the true expectation. In practice the algorithm is applied with $p$ replaced by the empirical distribution of the $N$ particles, and $\hat{h}$ replaced by the empirical average. One such finite- $N$ algorithm appears in Sec. 4. A rigorous convergence analysis for the finite- $N$ algorithm is currently an open problem and the subject of future work. For conventional particle filters, the convergence theory is by now welldeveloped; cf., $[37,51,11,13,12]$. It is likely that the techniques used in prior work can be extended to the present setting. One challenge is to account for the approximation of the solution to the boundary value problem BVP (4) that determines the filter gain.

\section{Well-posedness, Admissibility and Numerics}

\subsection{Weak formulation of the Poisson equation}

A function $\phi_{j} \in H_{0}^{1}\left(\mathbb{R}^{d} ; p\right)$ is said to be a weak solution of the BVP (4) if

$\int \nabla \phi_{j}(x, t) \cdot \nabla \psi(x) p(x, t) \mathrm{d} x=\int\left(h_{j}(x)-\hat{h}_{j}\right) \psi(x) p(x, t) \mathrm{d} x$,

for all $\psi \in H^{1}\left(\mathbb{R}^{d} ; p\right)$.

Denoting $\mathrm{E}[\cdot]:=\int \cdot p(x, t) \mathrm{d} x$, the weak form of the BVP (4) is expressed as

$$
\mathrm{E}\left[\nabla \phi_{j} \cdot \nabla \psi\right]=\mathrm{E}\left[\left(h_{j}-\hat{h}_{j}\right) \psi\right], \quad \forall \psi \in H^{1}\left(\mathbb{R}^{d} ; p\right) .
$$

This representation is also useful for the numerical algorithm described in Sec. 3.3.

\subsection{Main results on well-posedness and admissibility}

The existence-uniqueness result for the BVP (4) is described next - Its proof is given in Appendix A.2.

Theorem 2 Under Assumptions A1-A2, the BVP (4) possesses a unique weak solution $\phi_{j} \in H_{0}^{1}\left(\mathbb{R}^{d} ; p\right)$, satisfying

$$
\int\left|\nabla \phi_{j}(x)\right|^{2} p(x, t) \mathrm{d} x \leq \frac{1}{\lambda} \int\left|h_{j}(x)-\hat{h}_{j}\right|^{2} p(x, t) \mathrm{d} x .
$$

If in addition Assumption A3 holds, then the weak solution has higher regularity: $\phi_{j} \in H^{2}\left(\mathbb{R}^{d} ; p\right)$ with

$$
\int\left|D^{2} \phi_{j}\right|^{2} p(x, t) \mathrm{d} x \leq C(\lambda ; p) \int\left|\nabla h_{j}\right|^{2} p(x, t) \mathrm{d} x
$$

where $\lambda$ is (spectral gap) constant (see Assumption A2) and $C(\lambda ; p)=\lambda^{-2}\left(\lambda+\left\|D^{2}(\log p)\right\|_{L^{\infty}}\right)$.

The apriori bounds (13)-(14) are used to show that the control input for the feedback particle filter is admissible. The proof appears in Appendix A.3.

Corollary 1 Suppose $\phi_{j}$ is the weak solution of the BVP (4) as described in Thm. 2. The gain function $\mathrm{K}$ is obtained using (5) and $u$ is given by (10). Then

$$
\begin{aligned}
\mathrm{E}\left[|\mathrm{K}|^{2}\right] & \leq \frac{1}{\lambda} \sum_{j=1}^{m} \int\left|h_{j}(x)-\hat{h}_{j}\right|^{2} p(x, t) \mathrm{d} x, \\
\mathrm{E}[|u|] & \leq \text { (const. }) \sum_{j=1}^{m} \int\left(\left|h_{j}(x)\right|^{2}+\left|\nabla h_{j}\right|^{2}\right) p(x, t) \mathrm{d} x,
\end{aligned}
$$

where the constant depends on both $\lambda$ and $p$. That is, the resulting control input is admissible.

Example 1 The Gaussian case. Consider $p(x, t)=$ $\frac{1}{(2 \pi)^{\frac{d}{2}}\left|\Sigma_{t}\right|^{\frac{1}{2}}} \exp \left(-\frac{1}{2}\left(x-\mu_{t}\right)^{T} \Sigma_{t}^{-1}\left(x-\mu_{t}\right)\right)$, where $x=$ $\left(x_{1}, x_{2}, \ldots, x_{d}\right)^{T}, \mu_{t}=\left(\mu_{1 t}, \mu_{2 t}, \ldots, \mu_{d t}\right)^{T}$ is the mean, $\Sigma_{t}$ is the covariance matrix, and the determinant $\left|\Sigma_{t}\right|>0$. The unique solution of the BVP (4) is given by,

$$
\phi_{j}(x, t)=\sum_{k=1}^{d}\left[\Sigma_{t} H_{t}^{T}\right]_{k j}\left(x_{k}-\mu_{k t}\right) .
$$

In this case, $\mathrm{K}=\nabla \phi=\Sigma_{t} H_{t}^{T}$ is the Kalman gain.

\subsection{Numerical algorithm for approximation of $\mathrm{K}$}

In this section, a Galerkin finite-element algorithm is described to construct an approximate solution of (11). Since there are $m$ uncoupled PDEs, without loss of generality, a scalar-valued observation, with $m=1$, is assumed in this section so that $\mathrm{K}=\nabla \phi$. The time $t$ is fixed. The explicit dependence on time is suppressed for notational ease (That is, $p(x, t)$ is denoted as $p(x), \phi(x, t)$ as $\phi(x)$ etc. $)$.

Following (12), the objective is to approximate the scalarvalued solution $\phi(x)$ such that

$$
\mathrm{E}[\nabla \phi \cdot \nabla \psi]=\mathrm{E}[(h-\hat{h}) \psi]
$$

holds for all $\psi \in H^{1}\left(\mathbb{R}^{d} ; p\right)$ where $\mathrm{E}[\cdot]:=\int_{\mathbb{R}^{d}} \cdot p(x) \mathrm{d} x$. 


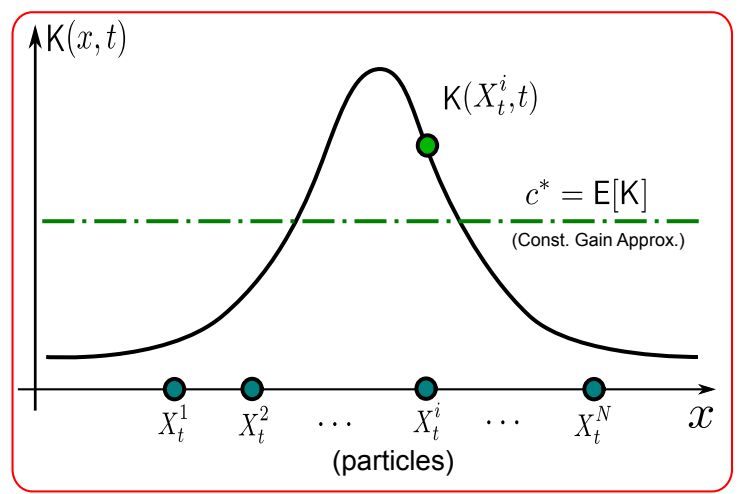

Fig. 2. Approximating nonlinear $\mathrm{K}$ by its expected value $\mathrm{E}[\mathrm{K}]$. For simplicity, the scalar case is depicted (i.e., $X_{t} \in \mathbb{R}$ ).

In the Galerkin approach, the function $\phi$ is approximated as,

$$
\phi(x)=\sum_{l=1}^{L} \kappa_{l} \psi_{l}(x)
$$

where $\left\{\psi_{l}(x)\right\}_{l=1}^{L}$ are a given set of basis functions. The gain function $\mathrm{K}$ is then given by

$$
\mathrm{K}(x)=\nabla \phi(x)=\sum_{l=1}^{L} \kappa_{l} \nabla \psi_{l}(x)
$$

The finite-dimensional approximation of (15) is to choose constants $\left\{\kappa_{l}\right\}_{l=1}^{L}$ such that

$$
\sum_{l=1}^{L} \kappa_{l} \mathrm{E}\left[\nabla \psi_{l} \cdot \nabla \psi\right]=\mathrm{E}[(h-\hat{h}) \psi], \quad \forall \psi \in S,
$$

where $S:=\operatorname{span}\left\{\psi_{1}, \psi_{2}, \ldots, \psi_{L}\right\} \subset H^{1}\left(\mathbb{R}^{d} ; p\right)$.

Denoting $[A]_{k l}=\mathrm{E}\left[\nabla \psi_{l} \cdot \nabla \psi_{k}\right], b_{k}=\mathrm{E}\left[(h-\hat{h}) \psi_{k}\right]$, and $\kappa=$ $\left(\kappa_{1}, \kappa_{2}, \ldots, \kappa_{L}\right)^{T}$, the finite-dimensional approximation (17) is expressed as a linear matrix equation:

$$
A \kappa=b .
$$

In a numerical implementation, the matrix $A$ and vector $b$ are approximated as,

$$
\begin{aligned}
{[A]_{k l} } & =\mathrm{E}\left[\nabla \psi_{l} \cdot \nabla \psi_{k}\right] \approx \frac{1}{N} \sum_{i=1}^{N} \nabla \psi_{l}\left(X_{t}^{i}\right) \cdot \nabla \psi_{k}\left(X_{t}^{i}\right), \\
b_{k} & =\mathrm{E}\left[(h-\hat{h}) \psi_{k}\right] \approx \frac{1}{N} \sum_{i=1}^{N}\left(h\left(X_{t}^{i}\right)-\hat{h}\right) \psi_{k}\left(X_{t}^{i}\right),
\end{aligned}
$$

where recall $\hat{h} \approx \frac{1}{N} \sum_{i^{\prime}=1}^{N} h\left(X_{t}^{i^{\prime}}\right)$. The important point to note is that the gain function is expressed in terms of averages taken over the population.
Example 2 Constant Gain Approximation. Suppose the basis functions are chosen to be the coordinate functions: $\psi_{k}(x)=x_{k}$ for $k=1,2, \ldots, d$. Writing $\psi(x)=$ $\left(\psi_{1}, \psi_{2}, \ldots, \psi_{d}\right)^{T}=x$,

$$
\begin{aligned}
\kappa=\mathrm{E}[\mathrm{K}] & =\mathrm{E}[(h-\hat{h}) \psi]=\int_{\mathbb{R}^{d}}(h(x)-\hat{h}) \psi(x) p(x) \mathrm{d} x \\
& \approx \frac{1}{N} \sum_{i=1}^{N} X_{t}^{i}\left(h\left(X_{t}^{i}\right)-\hat{h}\right) .
\end{aligned}
$$

This formula yields the constant gain approximation of the gain function.

Remark 4 Convergence in the Gaussian case. If $p$ is Gaussian and $h$ is linear, then, in the limit as $N \rightarrow \infty$, the constant gain approximation (20) equals the Kalman gain.

Remark 5 Variational interpretation. The constant gain approximation, formula (20), is the best - in the leastsquare sense - constant approximation of the gain function (see Fig. 2). Precisely, consider the following least-square optimization problem:

$$
c^{*}=\arg \min _{c \in \mathbb{R}^{d}} \mathrm{E}\left[|\mathrm{K}-c|^{2}\right] .
$$

By using a standard sum of square argument,

$$
c^{*}=\mathrm{E}[\mathrm{K}] \text {. }
$$

Likewise, the Galerkin solution (16) is the optimal leastsquare approximation in the finite-dimensional subspace $S$ of the infinite-dimensional Hilbert space $H^{1}\left(\mathbb{R}^{d} ; p\right)$.

Example 3 A sum-of-Gaussian scalar example. Consider a scalar (state dimension $d=1$ ) example, where the density is a sum of Gaussian,

$$
p(x)=\sum_{j=1}^{3} \lambda^{j} q^{j}(x)
$$

where $q^{j}(x)=q\left(x ; \mu^{j}, \Sigma^{j}\right)=\frac{1}{\sqrt{2 \pi \Sigma^{j}}} \exp \left(-\frac{\left(x-\mu^{j}\right)^{2}}{2 \Sigma^{j}}\right), \lambda^{j}>0$, $\sum_{j=1}^{3} \lambda^{j}=1$. The parameter values for $\lambda^{j}, \mu^{j}, \Sigma^{j}$ are tabulated in Fig. 3(a).

In the scalar case, a direct numerical solution (DNS) [56] of the gain function is obtained by numerically approximating the integral

$$
\mathrm{K}(x)=-\frac{1}{p(x)} \int_{-\infty}^{x}(h(y)-\hat{h}) p(y) \mathrm{d} y .
$$

The DNS solution is used to provide comparisons with the approximate Galerkin solutions. 


\begin{tabular}{c|c|c|c}
\multicolumn{4}{c}{} \\
\multicolumn{4}{c}{ Parameter Values } \\
\hline$j$ & $\lambda^{j}$ & $\mu^{j}$ & $\Sigma^{j}$ \\
\hline 1 & 0.2 & -1 & 0.25 \\
2 & 0.5 & 0 & 0.25 \\
3 & 0.3 & 1 & 0.25 \\
\hline
\end{tabular}

(a)

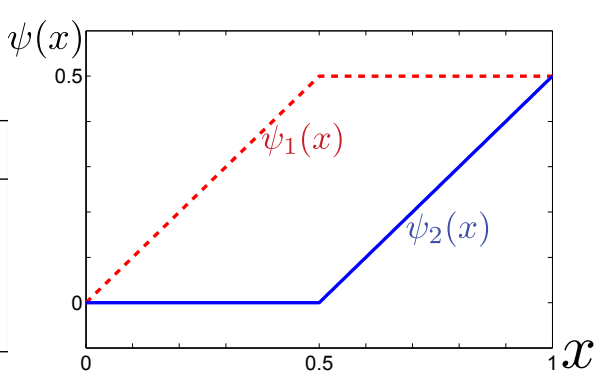

(b)
Fig. 3. (a) Parameter values and (b) basis functions $\left(\psi_{1}, \psi_{2}\right)$ in Example 3.

The Galerkin approximation of the gain function is constructed on an interval domain $D \subset \mathbb{R}$. The domain is a union of finitely many non-intersecting intervals $D_{k}=\left[a_{k-1}, a_{k}\right)$, where $k=1,2, \ldots, L$ and $a_{0}<a_{1}<\ldots<a_{L}$.

Define the basis functions as:

$$
\psi_{k}(x)=\left(x-a_{k-1}\right)_{+}-\left(x-a_{k}\right)_{+},
$$

where $f_{+}:=\frac{|f|+f}{2}$, so that,

$$
\nabla \psi_{k}(x)=1_{D_{k}}(x),
$$

where $1_{D_{k}}(x)$ denotes the indicator function with support on $D_{k}$.

As an example, Fig. 3(b) depicts the basis functions $\left\{\psi_{1}(x), \psi_{2}(x)\right\}$ for $D=[0,1]$ with $a_{0}=0, a_{1}=\frac{1}{2}$ and $a_{2}=1$. In this case, $\left\{\nabla \psi_{1}(x), \nabla \psi_{2}(x)\right\}$ are indicator functions on $\left[0, \frac{1}{2}\right)$ and $\left[\frac{1}{2}, 1\right)$.

Fig. 4 depicts a comparison of the DNS and Galerkin solution for $h(x)=x^{2}, D=[-2,2]$ and number of basis functions $L=1,5,15$. For a fixed $L$, the basis functions are constructed for a uniform partition of the domain (That is, $a_{l}=-2+\frac{l}{L} 4$ ). Two Galerkin approximations are considered in this example. One solution is obtained using $N=1000$ particles that are sampled from the density $p$ (see (21)). The particles are then used to compute matrix $A$ and vector $b$, according to formulae (18) and (19) respectively. Alternatively, since the analytical form of $p$ is known, these matrices can also be assembled by using the integrals:

$$
\begin{aligned}
{[A]_{k l} } & =\int \nabla \psi_{l}(x) \cdot \nabla \psi_{k}(x) p(x) \mathrm{d} x, \\
b_{k} & =\int(h(x)-\hat{h}) \psi_{k}(x) p(x) \mathrm{d} x .
\end{aligned}
$$

With $L=1$, the Galerkin solution is equivalent to the constant gain approximation method. As L increases, the Galerkin method gives a better approximation of the "true" nonlinear gain function (as approximated by the DNS). For $L=15$, the matrix $A$ was found to be singular for the particle-based implementation. This is because there are no particles in $D_{15}$. In this case, the Galerkin solution is obtained using only the integral formulae (22)-(23). These formulae are exact while the particle-based formulae (18) and (19) are approximations.

Note that the scalar example is included here only to illustrate the use of Galerkin method. Its extension to multivariate case is straightforward and the algorithm is summarized in Sec. 4.

\section{Algorithm summary and the linear Gaussian case}

\subsection{Finite-N FPF algorithm}

In a numerical implementation, one simulates $N$ particles according to the SDE:

$$
\begin{aligned}
\mathrm{d} X_{t}^{i} & =A_{t} X_{t}^{i} \mathrm{~d} t+\sigma_{t} \mathrm{~d} B_{t}^{i} \\
& +\mathrm{K}\left(X_{t}^{i}, t\right) \circ\left(\mathrm{d} Z_{t}-\frac{h\left(X_{t}^{i}\right)+\frac{1}{N} \sum_{j=1}^{N} h\left(X_{t}^{j}\right)}{2} \mathrm{~d} t\right),
\end{aligned}
$$

with initial conditions $X_{0}^{i}$ sampled i.i.d from the prior density $p^{*}(\cdot, 0)$. There are a number of standard approaches (e.g., Euler and Milstein schemes) for time-discretization and simulation of a Stratonovich SDE; cf., [29, Chapter 10]. The non-standard aspect of simulating (24) is approximation of the gain function $\mathrm{K}\left(X_{t}^{i}, t\right)$ at each discrete time-step. Finite- $N$ Galerkin algorithm for this purpose appears as Algorithm 1. In many applications, the computationally efficient constant gain approximation algorithm may suffice. This algorithm is described as Algorithm 2.

The choice of basis functions for the Galerkin approximation is problem-dependent. The constant-gain approximation corresponds to the choice of linear basis functions. An obvious extension is to consider quadratic or higher-order polynomial basis functions. Such basis functions are used, e.g., in the target tracking problem in Sec. 5.1. For problems with symmetry, the basis functions may be selected based on symmetry considerations. For example, circular basis functions are used for certain orbital tracking problems in [6], and the Fourier basis functions are used in $[50,49]$ for estimation of a stochastic process (phase) evolving on a circle.

The FPF algorithm presented in Sec. 1 represents the meanfield limit of the finite- $N$ algorithm. A rigorous analysis of the convergence properties of the finite- $N$ algorithm, based on propagation of chaos literature [47,35,27], is a subject of future work.

\subsection{Linear Gaussian case}

Consider the linear system,

$$
\begin{aligned}
& \mathrm{d} X_{t}=A_{t} X_{t} \mathrm{~d} t+\sigma_{t} \mathrm{~d} B_{t} \\
& \mathrm{~d} Z_{t}=H_{t} X_{t} \mathrm{~d} t+\mathrm{d} W_{t}
\end{aligned}
$$



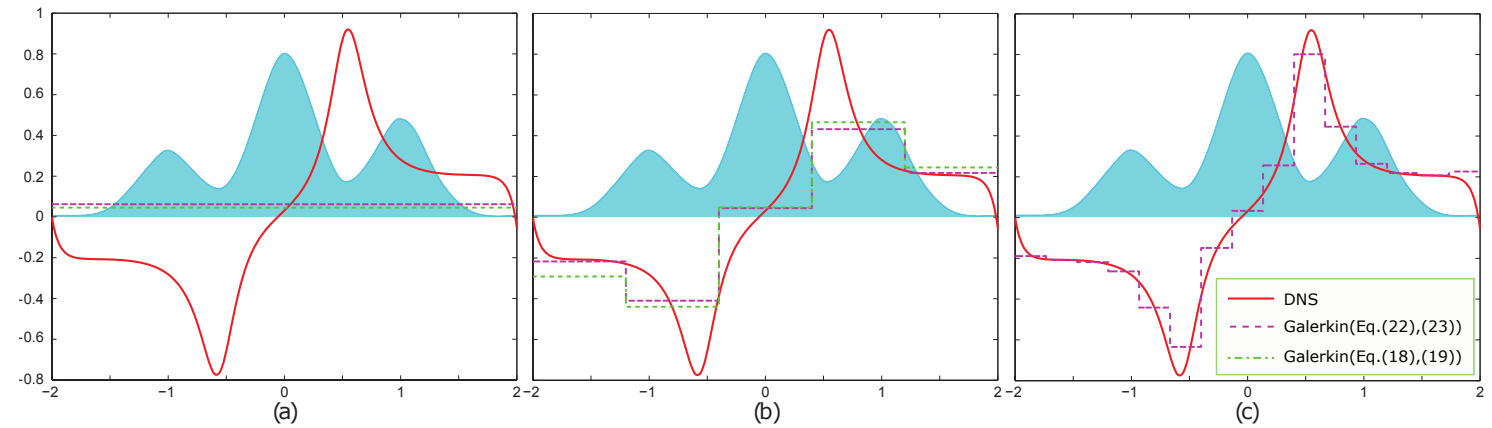

Fig. 4. Comparison of the DNS and the Galerkin approximations of the gain function for $h(x)=x^{2}$ and: (a) $L=1$, (b) $L=5$ and (c) $L=15$. The density is depicted as the shaded curve in the background.
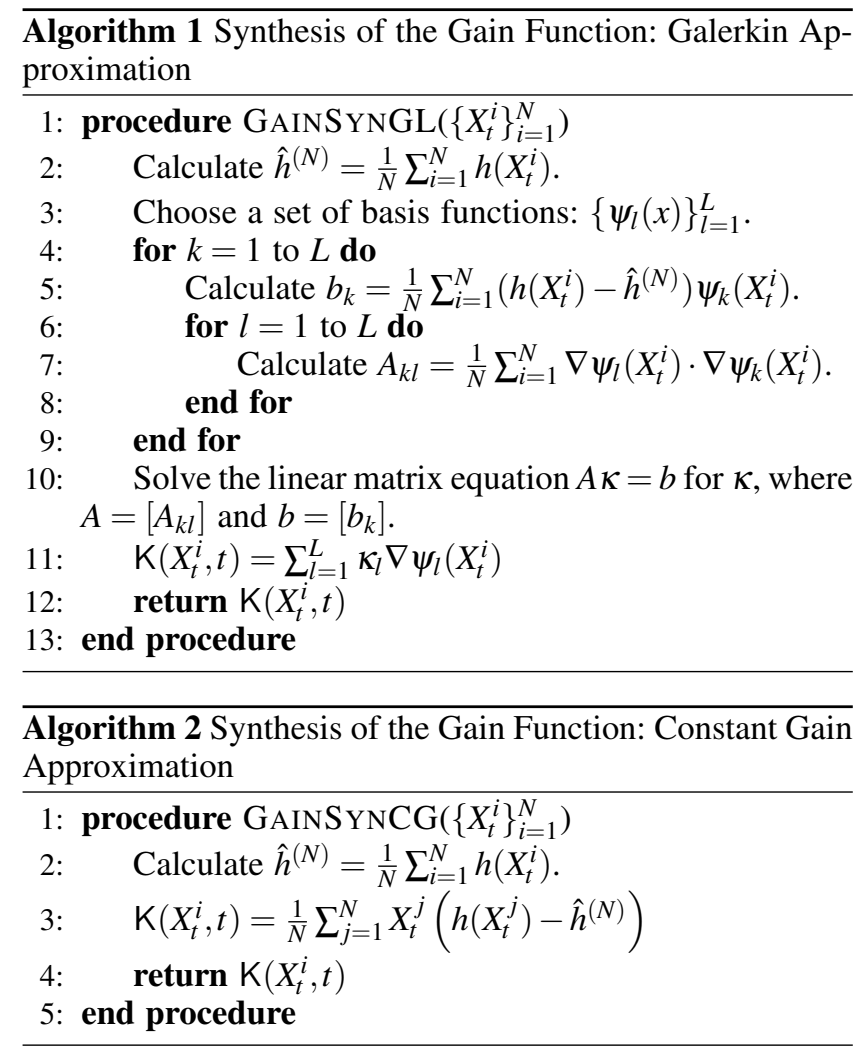

where, for any $t \geq 0, A_{t}, \sigma_{t}$ and $H_{t}$ are $d \times d, d \times d$ and $m \times d$ matrices, respectively. The initial density $p^{*}(x, 0)$ is Gaussian with mean vector $\mu_{0}$ and covariance matrix $\Sigma_{0}$.

For the Gaussian density $p(x, t)=(2 \pi)^{-\frac{d}{2}}\left|\Sigma_{t}\right|^{-\frac{1}{2}} \exp \left[-\frac{1}{2}(x-\right.$ $\left.\left.\mu_{t}\right)^{T} \Sigma_{t}^{-1}\left(x-\mu_{t}\right)\right]$, the exact solution of the BVP (4) is given in Example 1. As noted in Example 1, the gain function is the Kalman gain. This yields the following form for the linear Gaussian feedback particle filter:

$$
\mathrm{d} X_{t}^{i}=A_{t} X_{t}^{i} \mathrm{~d} t+\sigma_{t} \mathrm{~d} B_{t}^{i}+\Sigma_{t} H_{t}^{T}\left(\mathrm{~d} Z_{t}-H_{t} \frac{X_{t}^{i}+\mu_{t}}{2} \mathrm{~d} t\right)
$$

It is straightforward to verify that $p=p^{*}$ in this case. That is, the conditional density of $X_{t}$ and $X_{t}^{i}$ coincide, and are de- fined by the well-known dynamic equations that characterize the mean and covariance of the continuous-time KalmanBucy filter. The proof is a special case of Thm. 1 and hence omitted.

Theorem 3 Consider the linear Gaussian filtering problem defined by the state-observation equations (25a)-(25b). In this case the posterior densities of $X_{t}$ and $X_{t}^{i}$ are Gaussian, whose conditional mean and covariance are given by the respective $S D E$ and the $O D E$,

$$
\begin{aligned}
\mathrm{d} \mu_{t} & =A_{t} \mu_{t} \mathrm{~d} t+\Sigma_{t} H_{t}^{T}\left(\mathrm{~d} Z_{t}-H_{t} \mu_{t} \mathrm{~d} t\right), \\
\frac{\mathrm{d}}{\mathrm{d} t} \Sigma_{t} & =A_{t} \Sigma_{t}+\Sigma_{t} A_{t}^{T}+\sigma_{t} \sigma_{t}^{T}-\Sigma_{t} H_{t}^{T} H_{t} \Sigma_{t} .
\end{aligned}
$$

In practice $\left\{\mu_{t}, \Sigma_{t}\right\}$ are approximated as sample mean and sample covariance matrix from the ensemble $\left\{X_{t}^{i}\right\}_{i=1}^{N}$ :

$$
\begin{aligned}
& \mu_{t} \approx \mu_{t}^{(N)}:=\frac{1}{N} \sum_{i=1}^{N} X_{t}^{i} \\
& \Sigma_{t} \approx \Sigma_{t}^{(N)}:=\frac{1}{N-1} \sum_{i=1}^{N}\left(X_{t}^{i}-\mu_{t}^{(N)}\right)\left(X_{t}^{i}-\mu_{t}^{(N)}\right)^{T} .
\end{aligned}
$$

The resulting equation (26) for the $i$-th particle is given by

$$
\mathrm{d} X_{t}^{i}=A_{t} X_{t}^{i} \mathrm{~d} t+\sigma_{t} \mathrm{~d} B_{t}^{i}+\mathrm{K}_{t}^{(N)}\left(\mathrm{d} Z_{t}-H_{t} \frac{X_{t}^{i}+\mu_{t}^{(N)}}{2} \mathrm{~d} t\right)
$$

where the gain $\mathrm{K}_{t}^{(N)}=\Sigma_{t}^{(N)} H_{t}^{T}$. Alternatively, one can use the constant gain approximation formula (20) to approximate the gain directly. Note that the two gains are identical in this case.

As $N \rightarrow \infty, \mu_{t}^{(N)} \rightarrow \mu_{t}, \Sigma_{t}^{(N)} \rightarrow \Sigma_{t}$, and (26) represents the mean-field limit of the finite- $N$ particle system (27). As a result, the empirical distribution of the particle system approximates the posterior distribution (density) $p^{*}(x, t)$ (See also Remark 3). 


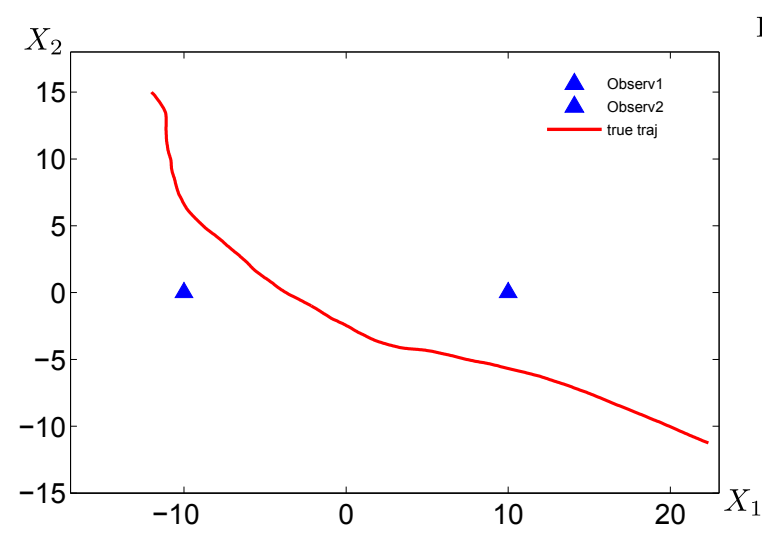

(a)

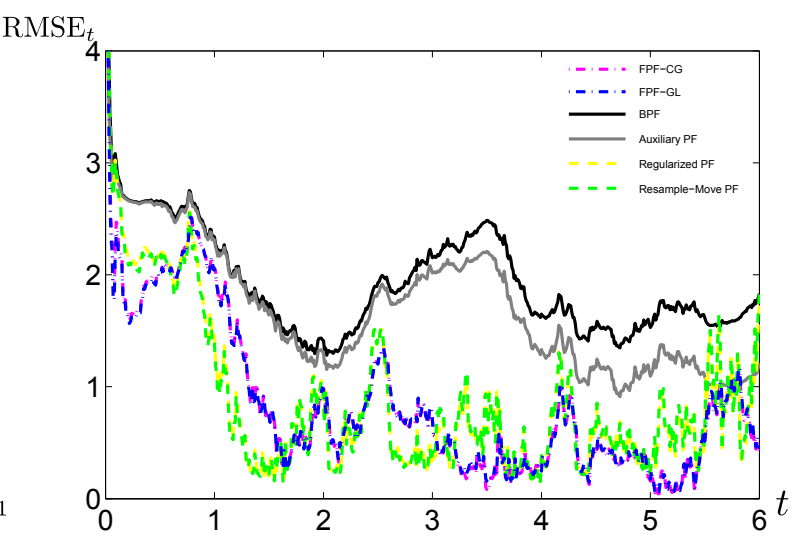

(b)

Fig. 5. Simulation results: (a) A sample trajectory of the target; (b) Comparison of RMSE with different particle filtering algorithms as a function of $t$, where $\sigma_{B}=1$.

\subsection{Comparison to the ensemble Kalman filter}

The linear FPF algorithm (27) is closely related to the Ensemble Kalman Filter (EnKF) [18]. The EnKF is an efficient numerical algorithm to approximate the Kalman filter for high dimensional systems. For such systems, the computational bottleneck in simulating a Kalman filter arises due to propagation of the covariance matrix according to the dynamic Riccati equation. This computation scales as $O\left(d^{3}\right)$ in memory where $d$ is the state dimension. In an EnKF implementation, one replaces the exact propagation of the covariance matrix by an empirical approximation with particles. This approximate computation scales as $O(N d)$ where $N$ is the number of particles. Using the notation of this paper, the resulting continuous-time EnKF is of the following form:

$\mathrm{d} X_{t}^{i}=A_{t} X_{t}^{i} \mathrm{~d} t+\sigma_{t} \mathrm{~d} B_{t}^{i}+\Sigma_{t}^{(N)} H_{t}^{T}\left(\mathrm{~d} Z_{t}-H_{t} X_{t}^{i} \mathrm{~d} t\right)$

Certain numerical techniques such as localization and ensemble inflations are typically used to improve the robustness with respect to sampling errors and non-Gaussian densities [33].

Both EnKF (28) and the linear FPF (27) possess an errorbased feedback control structure with identical approximation of the gain. In contrast to the conventional particle filtering approaches, the particles have equal weights and resampling is not necessary. Apart from the similarities, there are also important differences due to the difference in the form of the innovation error. In contrast to FPF, the EnKF algorithm (28) does not give the correct posterior in the limit as $N \rightarrow \infty$ : The SDE for the mean is the same as the Kalman filter. However, the equation for the variance is not correct for the EnKF. The major difference though is that linear FPF represents a special case of a more general algorithmic approach that is applicable to the general nonlinear non-Gaussian filtering problems.

Remark 6 The control term in the linear FPF (27) appears also in the work of Reich et. al [5], where it is referred to as the Ensemble Kalman-Bucy (EnKBF) filter. Note that the computational complexity of the linear FPF also scales as $O(N d)$.

\section{Numerics}

In this section, two numerical studies are presented to help illustrate and compare the performance of the multivariable FPF: i) a bearing-only target tracking problem in Sec. 5.1, and ii) a filtering problem for a high-dimensional linear Gaussian system in Sec. 5.2. Sec. 5.3 includes a short survey of some of the other numerical studies that provide comparison between the FPF and conventional particle filters.

\subsection{Bearing-only tracking example}

Consider a target tracking problem with bearing-only measurements [3,23]. A single target moves in a twodimensional (2d) plane according to the standard secondorder model:

$$
\mathrm{d} X_{t}=A X_{t} \mathrm{~d} t+\Gamma \mathrm{d} B_{t},
$$

where $X:=\left(X_{1}, V_{1}, X_{2}, V_{2}\right)^{T} \in \mathbb{R}^{4},\left(X_{1}, X_{2}\right)$ denotes the position and $\left(V_{1}, V_{2}\right)$ denotes the velocity. The matrices,

$$
A=\left[\begin{array}{llll}
0 & 1 & 0 & 0 \\
0 & 0 & 0 & 0 \\
0 & 0 & 0 & 1 \\
0 & 0 & 0 & 0
\end{array}\right], \quad \Gamma=\sigma_{B}\left[\begin{array}{ll}
0 & 0 \\
1 & 0 \\
0 & 0 \\
0 & 1
\end{array}\right],
$$

and $\left\{B_{t}\right\}$ is a standard $2 \mathrm{~d}$ Wiener process.

Two fixed sensors take bearing measurements $Z_{t}$ of the target according to the observation model,

$$
\mathrm{d} Z_{t}=h\left(X_{t}\right) \mathrm{d} t+\sigma_{W} \mathrm{~d} W_{t}
$$

where $\left\{W_{t}\right\}$ is a standard $2 \mathrm{~d}$ Wiener process, $h=\left(h_{1}, h_{2}\right)^{T}$ 


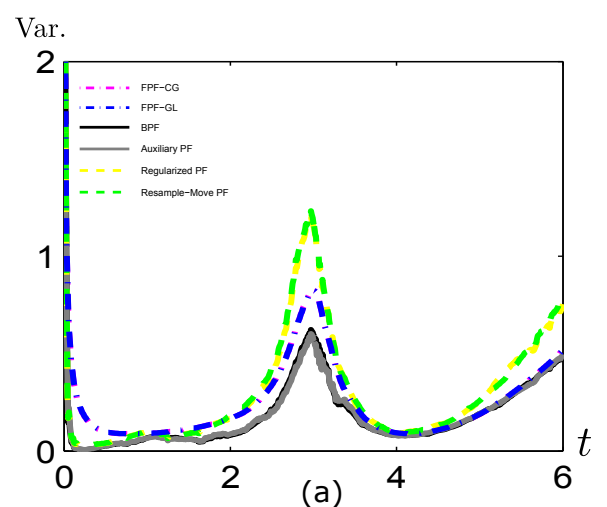

(a)

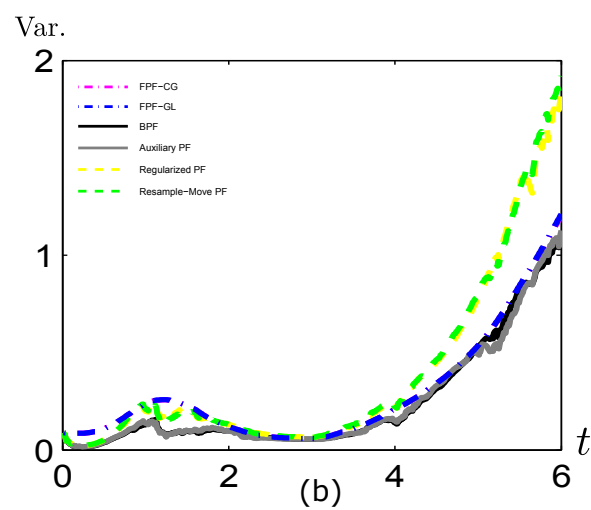

(b)

Fig. 6. Comparison of conditional variance of $X_{1}$ (a) and $X_{2}$ (b) estimated by different particle filtering algorithms, with $\sigma_{B}=1$. and

$$
h_{j}\left(x_{1}, v_{1}, x_{2}, v_{2}\right)=\arctan \left(\frac{x_{2}-x_{2}^{(\operatorname{sen} j)}}{x_{1}-x_{1}^{(\operatorname{sen} j)}}\right), \quad j=1,2,
$$

where $\left(x_{1}^{(\operatorname{sen} j)}, x_{2}^{(\operatorname{sen} j)}\right)$ denotes the position of sensor $j($ See Fig. 5(a)).

The following particle filter (PF) algorithms are implemented for comparison:

(1) FPF-CG: the FPF using the constant-gain approximation, as described in Algorithm 2.

(2) FPF-GL: the FPF using a Galerkin approximation, as described in Algorithm 1. Polynomial basis functions are used: $\left\{X_{1}, V_{1}, X_{2}, V_{2}, X_{1}^{2}, V_{1}^{2}, X_{2}^{2}, V_{2}^{2}\right\}$.

(3) BPF: the bootstrap PF as described in Ch. 9 of [2].

(4) APF: the auxiliary PF as described in [41].

(5) RPF: the regularized PF with an Epanechnikov kernel, as described in [38].

(6) RMPF: the resample-move PF with a MetropolisHastings MCMC move step, as described in [21].

Note the BPF algorithm, also referred to Sampling Importance Resampling filter, is one of the most widely used PF algorithms. The BPF uses the prior density as the importance density and applies the systematic resampling procedure [28] at each discrete time step. Other conventional PF algorithms evaluated in this study differ from the BPF algorithm in the choice of importance density and resampling procedure.

The performance metric is the position root-mean-squared error (RMSE) over time $t$ :

$$
\operatorname{RMSE}_{\mathrm{t}}=\sqrt{\frac{1}{M} \sum_{j=1}^{M}\left(\left(\hat{X}_{1}\right)_{t}^{j}-\left(X_{1}\right)_{t}\right)^{2}+\left(\left(\hat{X}_{2}\right)_{t}^{j}-\left(X_{2}\right)_{t}\right)^{2}}
$$

where $\left(\hat{X}_{1}\right)_{t}^{j}$ denotes the estimated mean (approximated by particles) of $X_{1}$ at time $t$ for the $j$-th Monte Carlo run, $j=$ $1, \ldots, M$, and $\left(X_{1}\right)_{t}$ is the true simulated state. The metric is adopted because of its widespread use in the target tracking literature $[23,1,15]$.

The simulation parameters are as follows: The true initial state of the target is $(-12,4,15,-5)^{T}$, and the signal noise level $\sigma_{B}=1$; Two sensors are located at fixed points $(-10,0)^{T}$ and $(10,0)^{T}$, and $\sigma_{W}=0.017$. The simulation is carried out over a finite time-horizon $t \in[0, T]$ with $T=6$ and a fixed discretized time-step $\Delta t=0.01$. All PFs use $N=500$ particles and have the same initialization, where particles are drawn from a multivariate Gaussian distribution with mean vector $\mu_{0}=(-5,3,14,-6)^{T}$ and covariance matrix $\Sigma_{0}=\operatorname{diag}\left(\left[1.5^{2}, 0.05^{2}, 0.3^{2}, 0.01^{2}\right]\right)$. A total number of $M=100$ Monte Carlo simulation runs are performed to obtain the results.

Fig. 5(a) depicts a sample path of the target trajectory for a typical simulation run. Fig. 5(b) compares the $\mathrm{RMSE}_{t}$ for six PFs. Table 1 lists the time-averaged RMSE with different signal noise levels $\left(\sigma_{B} \in\{0.5,1,2\}\right)$. The timeaveraged RMSE is defined as:

RMSE $=$

$\sqrt{\frac{1}{T} \int_{t=0}^{T}\left[\frac{1}{M} \sum_{j=1}^{M}\left(\left(\hat{X}_{1}\right)_{t}^{j}-\left(X_{1}\right)_{t}\right)^{2}+\left(\left(\hat{X}_{2}\right)_{t}^{j}-\left(X_{2}\right)_{t}\right)^{2}\right] \mathrm{d} t .}$

The mean computation time (in milliseconds, for a single update step) is also provided for the case $\sigma_{B}=1$. It is measured with MATLAB tic toc function on an Intel i5 $2.5 \mathrm{GHz}$ CPU, 8GB RAM platform.

It is shown in Table 1 that the FPF-CG and FPF-GL have the best performance in terms of RMSE, consistently over different noise levels. In this particular example, the FPFCG and FPF-GL have comparable performance, while the FPF-CG is much faster than FPF-GL and other PFs.

Since there is no ground truth for the conditional variance for the nonlinear tracking problem, Fig. 6 provides a comparison of the estimated variance for $X_{1}$ and $X_{2}$, using the six PFs. It is seen that, for this particular example, the regularized $\mathrm{PF}$ and resample-move PF have the largest variance, BPF and 

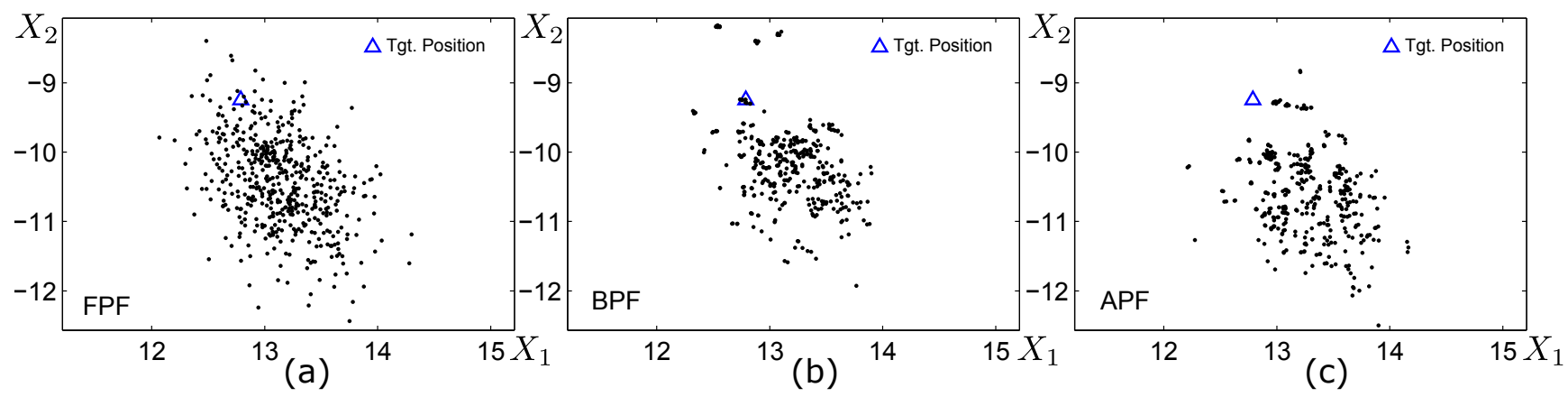

Fig. 7. Scatterplots of 500 particle samples on the $X_{1}-X_{2}$ plane from the FPF (a), BPF (b) and APF(c).

Table 1

Performance comparison

\begin{tabular}{|c|c|c|c|c|}
\hline & $\sigma_{B}=0.5$ & $\sigma_{B}=1$ & $\sigma_{B}=2$ & Comp. Time \\
\hline \hline FPF-CG & 0.90 & 0.93 & 1.03 & 7 \\
\hline FPF-GL & 0.90 & 0.94 & 1.03 & 265 \\
\hline BPF & 1.77 & 1.28 & 1.24 & 25 \\
\hline APF & 1.92 & 1.24 & 1.18 & 54 \\
\hline RPF & 0.95 & 0.98 & 1.09 & 25 \\
\hline RMPF & 0.97 & 1.00 & 1.10 & 75 \\
\hline
\end{tabular}

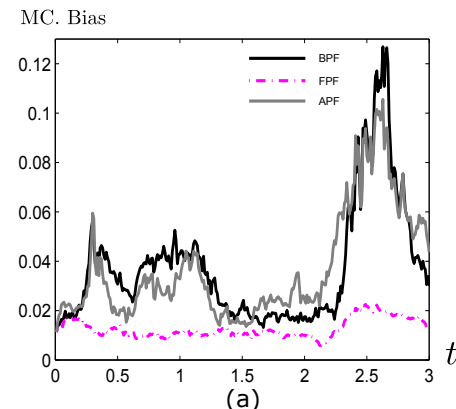

(a)

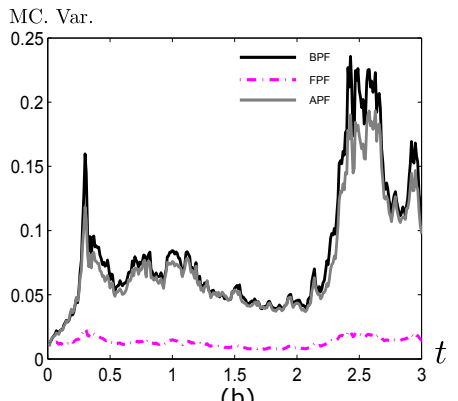

(b)

APF have the lowest, and FPF-CG and FPF-GL estimates lie in the middle. All PFs have a similar trend for the estimated conditional variance.

As the signal noise decreases, the performance of BPF and APF degenerates as a result of particle impoverishment, which essentially means the particle population lose its diversity because particles with high weights are statistically selected many times in the resampling step [1]. Fig. 7 depicts a snapshot (at $t=6$ ) of the scatterplot of particles using the FPF, BPF and APF, respectively. The true target position is depicted as a triangle. Although the point estimates of all algorithms are close, the FPF provides a 'smoother' characterization of the posterior distribution. More advanced PF algorithms, such as RPF and RMPF, are able to alleviate particle improvishment and improve estimation accuracy, as is shown in Table 1 . However, these approaches usually require a careful design of the kernel density (RPF) or a good choice of the Markov transition kernel (RMPF). This is often difficult in large scale systems.

Remark 7 Computational complexity. By implementing the Bayes' rule via an innovation error based feedback control structure, the FPF avoids the importance sampling and resampling step of the conventional PF algorithms. As a result, the FPF does not adversely suffer from samplingrelated issues, such as particle impoverishment and particle degeneracy. The computational bottleneck in FPF arises because of the BVP (4) which needs to be solved at each time step to compute the gain function. In practice, FPF-CG with the constant gain approximation is a computationally attractive option. For certain problems with severe nonlinearity, it has been shown that the FPF-GL can significantly

Fig. 8. Monte Carlo bias (a) and variance (b) for the estimated mean by the FPF, BPF and APF.

outperform both FPF-CG and other PFs [6]. However, the improved accuracy comes with a cost of additional computational cost. Computationally efficient gain approximation techniques is a subject of continuing work.

\subsection{Filtering of a high-dimensional system}

It has been noted in literature that the conventional PFs (based on importance sampling) may fail in large scale systems [4]. To shed light on the effects of dimensionality on filter performance, we provide here a comparison between the FPF, BPF and APF with respect to the estimation error. Consider a linear Gaussian example:

$$
\begin{aligned}
& \mathrm{d} X_{t}=-0.5 X_{t} \mathrm{~d} t+\mathrm{d} B_{t}, \\
& \mathrm{~d} Z_{t}=X_{t} \mathrm{~d} t+\mathrm{d} W_{t}, \quad X_{0} \sim N\left(0, I_{d}\right),
\end{aligned}
$$

where $X_{t}$ is the $d$-dimensional state vector, $Z_{t}$ is the $d$ dimensional observation vector, $I_{d}$ denotes the $d \times d$ identity matrix and $\left\{B_{t}\right\},\left\{W_{t}\right\}$ are independent standard Wiener processes with covariance $I_{d}$. The prior distribution is assumed to be Gaussian with zero mean and covariance matrix $\Sigma_{0}=I_{d}$. These choices allow for a straightforward manipulation of system parameters for simulation and comparison purposes.

For the linear Gaussian filtering problem (30)-(31), the optimal solution is given by the Kalman-Bucy filter [2]. In the following, five numerical studies are conducted. For each case, a total of $M=100$ Monte Carlo runs are performed to obtain the results reported here. For each simulation run, a 

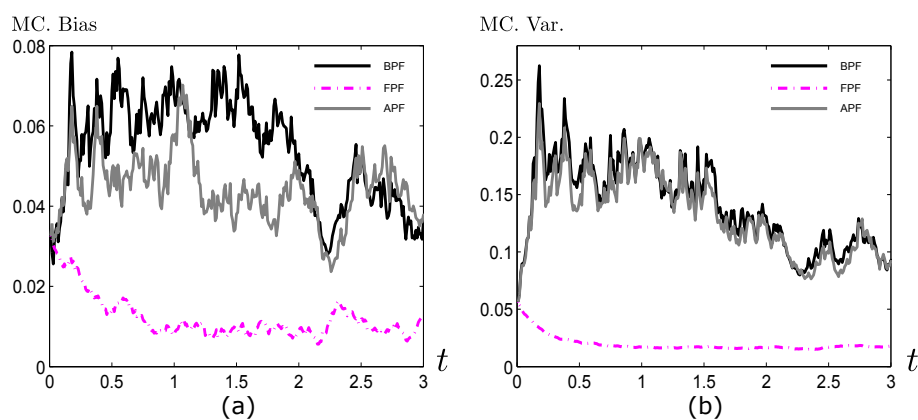

Fig. 9. Monte Carlo bias (a) and variance (b) for the estimated covariance by the FPF, BPF and APF.

fixed discrete time-step of $\Delta t=0.01$ and a total simulation time of $T=3$ are used. The FPF uses the constant gain approximation for its gain calculation (Algorithm 2), the BPF is implemented according to Ch. 9 of [2] with systematic resampling, and the APF implementation is based on the algorithm provided in [1]. All filters have the same initialization, where the initial particles are drawn from the standard $d$-dimensional Gaussian distribution.

(1) Monte Carlo bias and variance for the estimated mean: Define the estimation error of particle filters in terms of Monte Carlo bias and variance:

$$
\begin{aligned}
\text { M. C. } \operatorname{Bias}\left(\mu_{t}^{(N)}\right) & =\left|\frac{1}{M} \sum_{j=1}^{M}\left(\mu_{t, j}^{(N)}-\mu_{t}\right)\right|, \\
\text { M. C. } \operatorname{Var}\left(\mu_{t}^{(N)}\right) & =\frac{1}{M} \sum_{j=1}^{M}\left|\mu_{t, j}^{(N)}-\frac{1}{M} \sum_{j=1}^{M} \mu_{t, j}^{(N)}\right|^{2},
\end{aligned}
$$

where $\mu_{t}$ is the true conditional mean vector given by the Kalman-Bucy filter, $\mu_{t}^{(N)}$ is the estimated mean computed by particle filters, $\mu_{t, j}^{(N)}$ is the PF estimate in the $j$-th Monte Carlo run, and $|\cdot|$ denotes the Euclidean norm. In the simulation, we set the dimension $d=$ 5 and use a fixed number of particles $N=500$. The results are depicted in Fig. 8. Note that the FPF estimate consistently outperforms the BPF and APF with respect to both Monte Carlo bias and variance.

(2) Monte Carlo bias and variance for the estimated covariance: Define the Monte Carlo bias and variance for the covariance matrix estimate as follows:

$$
\begin{aligned}
\text { M. C. } \operatorname{Bias}\left(\Sigma_{t}^{(N)}\right) & =\left\|\frac{1}{M} \sum_{j=1}^{M}\left(\Sigma_{t, j}^{(N)}-\Sigma_{t}\right)\right\|_{F}, \\
\text { M. C. } \operatorname{Var}\left(\Sigma_{t}^{(N)}\right) & =\frac{1}{M} \sum_{j=1}^{M}\left\|\Sigma_{t, j}^{(N)}-\frac{1}{M} \sum_{j=1}^{M} \Sigma_{t, j}^{(N)}\right\|_{F}^{2},
\end{aligned}
$$

where $\Sigma_{t}$ is the true conditional covariance matrix given by the Kalman-Bucy filter, $\Sigma_{t}^{(N)}$ is the estimated covariance matrix coumputed by particle filters, $\Sigma_{t, j}^{(N)}$ is the PF estimate in the $j$-th Monte Carlo run, and $\|\cdot\|_{F}$ denotes the (matrix) Frobenius norm. In the simulation,
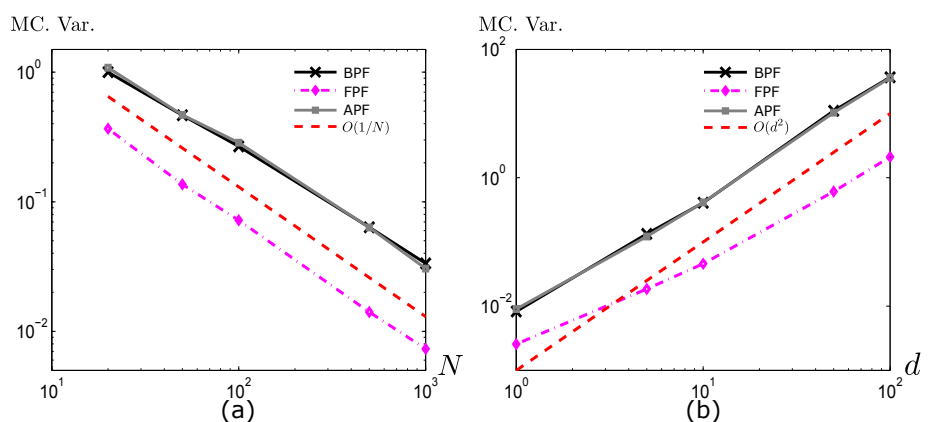

Fig. 10. Comparisons of the Monte Carlo variance for the FPF, $\mathrm{BPF}$ and APF as a function of: (a) $N$; (b) $d$.

we set the dimension $d=5$ and use a fixed number of particles $N=500$. The result appears in Fig. 9. Note that the FPF estimate has consistently smaller Monte Carlo bias and variance than the BPF and APF.

(3) Monte Carlo variance as a function of the number of particles $N$ : In this simulation, the dimension is set to $d=5$ and $N$ is taken from a set of $\{20,50,100,500,1000\}$. The filter performance is compared in terms of Monte Carlo variance for the estimated mean, over $N$, as depicted in Fig. 10(a). While the Monte Carlo variance decreases as $O\left(\frac{1}{N}\right)$ as the number of particles increases, the FPF has a consistently smaller variance than the BPF. The $O(1 / N)$ line is included to aid the comparison.

(4) Monte Carlo variance as a function of the state dimension $d$ : In this simulation, the signal state dimension is taken from a set of $\{1,5,10,50,100\}$ and a fixed number of particles $N=500$ is used. The Monte Carlo variance for the estimated mean is calculated for each $d$ and the result appears in Fig. 10(b). The $O\left(d^{2}\right)$ line is included to aid the comparison. This simulation result suggests that the feedback can help reduce the high variance that is often observed with the conventional $\mathrm{PFs}$, especially when the underlying system is of high dimension (e.g., $d=100$ ). In this case, the BPF and APF suffer from particle degeneracy, i.e., the particle population collapses to a single mass point after only a few iterations. It has also been shown theoretically that the degeneracy phenomenon is impossible to avoid in importance sampling-based approaches [15]. The root cause for particle degeneracy is the pointwise multiplication implementation of Bayes' rule; cf., $[4,10,16]$. In the FPF algorithm, the particles are directly "migrated' to the desired regions of the state space by using a error correction-based feedback control approach, thereby avoiding the degeneracy issues.

(5) Computational time as a function of $N$ and $d$ : In this simulation, we first fix the dimension $d=5$ and let $N$ vary from $\{20,50,100,500,1000\}$. Then we fix $N=500$ and let $d$ vary from $\{1,5,10,50,100\}$. The mean computational time (per iteration cycle, averaged over 100 Monte Carlo runs) is depicted against $N$ and $d$ in Fig. 11(a) and (b), respectively. This result suggests that with a constant gain approximation, the FPF has a lower computational cost compared to the BPF and 

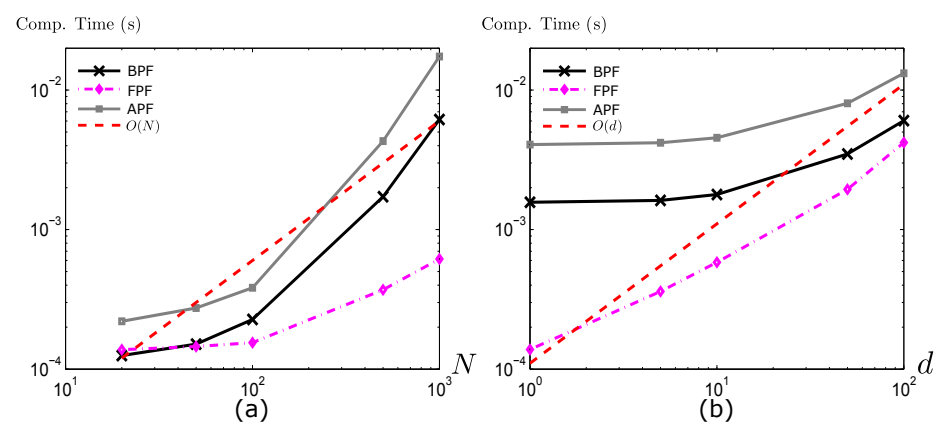

Fig. 11. Comparisons of the mean computational time for the FPF, BPF and APF as a function of: (a) $N$; (b) $d$.

APF. The $O(N)$ and $O(d)$ lines are included to aid the comparison.

\subsection{Additional numerical examples}

The following is a summary of other numerical studies that provide additional comparisons between the FPF and conventional PFs:

(1) In a recent paper by Berntorp [6], the FPF-CG and FPFGL are evaluated and compared against the Unscented Kalman filter (UKF), BPF, LLPF (a PF using optimal sampling with linearized likelihood [15]), and the RaoBlackwellized PF [43]. The comparisons are carried out for two target tracking example problems: A 'reentry' problem where a vehicle enters the atmosphere at high speed, and a 'two-body' problem with a satellite in a Keplerian orbit around earth. The measurements use bearing only sensors. The paper's conclusion is that the FPF-CG is more accurate than the other PFs without sacrificing computational efficiency. For the two-body problem, it is shown that the FPF-GL is able to significantly improve estimation accuracy compared to the FPF-CG and other PF algorithms.

(2) In Stano's PhD work at Delft $[44,45]$, extensive numerical experiments were conducted to compare the performance of various PF algorithms for the 'hopper dredger' nonlinear estimation problem. The FPF-CG is compared against the Reduced-Order PF (ROPF), BPF and improved Saturated PF (iSPF). The paper's conclusion is that the FPF "provides very accurate estimates with a low computational price" and "is robust [without the need for] tuning parameters".

(3) Tilton et. al [48] compare the FPF-CG to EKF and a range of $\mathrm{PF}$ algorithms with different resampling techniques on a benchmark nonlinear filtering problem from a tutorial paper [7]. For the example problem, it is shown that the FPF provides better or comparable performance at a fraction of the computational cost.

\section{Conclusions}

In this paper, we described the feedback particle filter (FPF) in the general multivariable setting. The FPF provides for a generalization of the Kalman filter to a general class of nonlinear non-Gaussian problems. The Bayes' rule is implemented via an innovation error-based feedback control structure, which is important on account of the issue of robustness. In particular, feedback can help reduce the high variance that is sometimes observed with the conventional particle filters, especially in large scale systems as a result of particle degeneracy. Numerical results are presented to support this claim (see Fig. 10).

The solution of the gain function is the central problem of the FPF algorithm. Motivated by a weak formulation of Poisson's equation, a Galerkin finite-element algorithm is proposed for approximation of the gain. Future research directions concerning the gain function approximation include the following:

- The choice of the optimal basis functions in the Galerkin approach. The performance of the FPF depends on the selection of basis functions and the best way to do this remains an important open problem.

- The recursive-form solution of the gain function. This involves the construction of a differential equation that describes the evolution of the gain function with time. Recall that the Galerkin method proposed in this paper is memoryless (i.e., it does not depend on the history).

- Approximations via relationship to MCMC. The Smoluchowski equation models a $d$-dimensional gradient flow with "noise":

$$
\mathrm{d} \Phi_{t}=-\nabla \mathscr{G}\left(\Phi_{t}\right) \mathrm{d} t+\sqrt{2} \mathrm{~d} \xi_{t}
$$

where $\xi$ is a standard Wiener process. It is regarded as the original MCMC algorithm: Under general conditions it is ergodic, with (unnormalized) stationary density $e^{-\mathscr{G}}$. The PDE (4) can be expressed as an instance of Poisson's equation for this diffusion,

$$
\mathscr{D} \phi_{j}=-\left(h_{j}-\hat{h}_{j}\right), \quad 1 \leq j \leq m,
$$

where $\mathscr{D}$ is the differential generator for the Smoluchowski equation, with potential $\mathscr{G}=-\log p$. Subject to growth conditions on $h$ and $p$, this implies the mean-integral representation for the function,

$$
\phi_{j}(x)=\int_{0}^{\infty} \mathrm{E}\left[h_{j}\left(\Phi_{t}\right)-\hat{h}_{j} \mid \Phi_{0}=x\right] \mathrm{d} t .
$$

This representation also suggests an alternate proof of well-posedness and construction of numerical algorithms; cf., [22].

\section{Acknowledgements}

Financial support from the NSF grants 1334987 and 1462773, and the Simons foundation grant 204296 is gratefully acknowledged. Comments from an anonymous reviewer helped substantially improve this paper. The reviewer's input is gratefully acknowledged. 


\section{A Appendix}

\section{A.1 Proof of Thm. 1}

Formally, the PDE (4) that the gain function has to satisfy can be written as:

$$
\nabla \cdot(p \mathrm{~K})=-(h-\hat{h})^{T} p
$$

where the divergence operator is applied to each column of the matrix $p \mathrm{~K}$. It is only necessary to show that with the choice of $\{u, \mathrm{~K}\}$ given by (A.1) and (10), we have $\mathrm{d} p(x, t)=$ $\mathrm{d} p^{*}(x, t)$, for all $x$ and $t$, in the sense that they are defined by identical stochastic differential equations. Recall $\mathrm{d} p^{*}$ is defined according to the K-S equation (7), and $\mathrm{d} p$ according to the forward equation (9).

Recall that the gain function $\mathrm{K}$ is a solution of the following PDE:

$$
\nabla \cdot(p \mathrm{~K})=-p(h-\hat{h})^{T} .
$$

On multiplying both sides of (10) by $-p$, we obtain

$$
\begin{aligned}
-u p & =\frac{1}{2} p \mathrm{~K}(h-\hat{h})-\Omega p+p \mathrm{~K} \hat{h} \\
& =-\frac{1}{2} \mathrm{~K}[\nabla \cdot(p \mathrm{~K})]^{T}-\Omega p+p \mathrm{~K} \hat{h}
\end{aligned}
$$

where (A.2) is used to obtain the second equality. Denoting $E:=\frac{1}{2} \mathrm{~K}[\nabla \cdot(p \mathrm{~K})]^{T}$, a direct calculation shows that

$$
E_{l}+\Omega_{l} p=\frac{1}{2} \sum_{k=1}^{d} \frac{\partial}{\partial x_{k}}\left(p\left[\mathrm{KK}^{T}\right] l k\right)
$$

Substituting this in (A.3), on taking the divergence of both sides, we obtain

$$
-\nabla \cdot(p u)+\frac{1}{2} \sum_{l, k=1}^{d} \frac{\partial^{2}}{\partial x_{l} \partial x_{k}}\left(p\left[\mathrm{KK}^{T}\right]_{l k}\right)=\nabla \cdot(p \mathrm{~K}) \hat{h}
$$

Using (A.2) and (A.4) in the forward equation (9),

$$
\mathrm{d} p=\mathscr{L}^{\dagger} p+(h-\hat{h})^{T}\left(\mathrm{~d} Z_{t}-\hat{h} \mathrm{~d} t\right) p
$$

This is precisely the SDE (7), as desired.

\section{A.2 Proof of Thm. 2}

We omit the subscript " $j$ " in this proof, writing just $\phi$ and $h$. We also suppress explicit dependence on time $t$, writing $p(x)$ instead of $p(x, t)$ and $\phi(x)$ instead of $\phi(x, t)$.

Under Assumption A2, $p$ satisfies the Poincaré inequality with constant $\lambda \geq c_{1}$ : That is, for all functions $\phi \in$
$H_{0}^{1}\left(\mathbb{R}^{d} ; p\right)$,

$$
\int|\phi(x)|^{2} p(x) \mathrm{d} x \leq \frac{1}{\lambda} \int|\nabla \phi(x)|^{2} p(x) \mathrm{d} x .
$$

Consider now the inner product

$$
<\phi, \psi>:=\int \nabla \phi(x) \cdot \nabla \psi(x) p(x) \mathrm{d} x
$$

On account of (A.5), the norm defined by using the inner product $\langle\cdot, \cdot\rangle$ is equivalent to the standard norm in $H^{1}\left(\mathbb{R}^{d} ; p\right)$.

(i) Consider the PDE in its weak form (11). The integral on the righthand-side is a bounded linear functional on $\psi \in H_{0}^{1}$, since

$$
\begin{aligned}
& \left|\int(h(x)-\hat{h}) \psi(x) p(x) \mathrm{d} x\right|^{2} \\
& \quad \leq \int|h(x)-\hat{h}|^{2} p(x) \mathrm{d} x \int|\psi(x)|^{2} p(x) \mathrm{d} x \\
& \quad \leq \text { (const.) } \int|\nabla \psi(x)|^{2} p(x) \mathrm{d} x,
\end{aligned}
$$

where (A.5) is used to obtain the second inequality.

It follows from the Reisz representation theorem that there exists a unique $\phi \in H_{0}^{1}$ such that

$$
<\phi, \psi>=\int(h(x)-\hat{h}) \psi(x) p(x) \mathrm{d} x
$$

for all $\psi \in H_{0}^{1}\left(\mathbb{R}^{d} ; p\right)$. The last formula holds also if we add any constant to $\psi$, and so it holds for all $\psi \in$ $H^{1}\left(\mathbb{R}^{d} ; p\right)$. Thus $\phi$ is a weak solution of the PDE, satisfying (11).

(ii) Suppose $\phi$ is a weak solution. Using $\psi=\phi$ in (11),

$$
\begin{aligned}
& \int|\nabla \phi|^{2} p(x) \mathrm{d} x=\int(h(x)-\hat{h}) \phi(x) p(x) \mathrm{d} x \\
& \leq\left(\int|h(x)-\hat{h}|^{2} p(x) \mathrm{d} x\right)^{\frac{1}{2}}\left(\int|\phi(x)|^{2} p(x) \mathrm{d} x\right)^{\frac{1}{2}} \\
& \leq\left(\int|h(x)-\hat{h}|^{2} p(x) \mathrm{d} x\right)^{\frac{1}{2}}\left(\frac{1}{\lambda} \int|\nabla \phi(x)|^{2} p(x) \mathrm{d} x\right)^{\frac{1}{2}}
\end{aligned}
$$

by (A.5). The estimate (13) follows.

(iii) For the final estimate (14), we establish the following bound:

Lemma 1 Under Assumptions A1-A3, the weak solution $\phi$ of the PDE (11) belongs to $H^{2}\left(\mathbb{R}^{d} ; p\right)$, with

$$
\int\left|D^{2} \phi\right|^{2} p \mathrm{~d} x \leq \int \nabla \phi \cdot G p \mathrm{~d} x
$$


where the vector function $G \in L^{2}\left(\mathbb{R}^{d} ; p\right)$ is defined by

$$
G=D^{2}(\log p) \nabla \phi+\nabla h
$$

and where $\left|D^{2} \phi\right|^{2}=\sum_{j, k}\left(\frac{\partial^{2} \phi}{\partial x_{j} \partial x_{k}}\right)^{2}$.

PROOF. First note that each entry of the Hessian matrix $D^{2}(\log p)$ is bounded, by Assumption A3, and that $\nabla h \in L^{2}\left(\mathbb{R}^{d} ; p\right)$ by Assumption A1. Hence $G \in$ $L^{2}(\mathbb{R} ; p)$.

Next, elliptic regularity theory $[17$, Section 6.3 of the PDE text by Evans] applied to the weak solution $\phi \in$ $H^{1}\left(\mathbb{R}^{d} ; p\right)$ says that $\phi \in H_{l o c}^{3}\left(\mathbb{R}^{d}\right)$. Hence the partial differential equation holds pointwise:

$$
-\nabla \cdot(p \nabla \phi)=(h-\hat{h}) p .
$$

Differentiating with respect to $x_{k}$ gives:

$$
\begin{aligned}
-\nabla \cdot\left(p \nabla \frac{\partial \phi}{\partial x_{k}}\right)-\nabla\left(\frac{\partial \log p}{\partial x_{k}}\right) \cdot(p \nabla \phi) \\
-\frac{\partial \log p}{\partial x_{k}} \nabla \cdot(p \nabla \phi)=\frac{\partial h}{\partial x_{k}} p+(h-\hat{h}) \frac{\partial \log p}{\partial x_{k}} p .
\end{aligned}
$$

The final terms on the left and right sides cancel, by equation (A.7). Thus the preceding formula becomes

$$
-\nabla \cdot\left(p \nabla \frac{\partial \phi}{\partial x_{k}}\right)=G_{k} p
$$

where $G_{k}$ denotes the $k$ th component of $G(x)$.

Let $\beta(x) \geq 0$ be a smooth, compactly supported "bump" function, meaning $\beta(x)$ is radially decreasing with $\beta(0)=1$. Let $s>0$ and multiply (A.8) by $\beta(s x)^{2} \frac{\partial \phi}{\partial x_{k}}$. Integrate by parts on the left side (noting the boundary terms vanish because $\beta$ has compact support) to obtain

$$
\int \nabla\left[\beta(s x)^{2} \frac{\partial \phi}{\partial x_{k}}\right] \cdot\left(\nabla \frac{\partial \phi}{\partial x_{k}}\right) p \mathrm{~d} x=\int \beta(s x)^{2} \frac{\partial \phi}{\partial x_{k}} G_{k} p \mathrm{~d} x .
$$

The right hand side RHS $\rightarrow \int \frac{\partial \phi}{\partial x_{k}} G_{k} p \mathrm{~d} x$ by dominated convergence as $s \rightarrow 0$, since $\beta(0)=1$. The left side of (A.9) can be expressed as:

$$
\begin{aligned}
\text { LHS } & =\int \beta(s x)^{2}\left|\nabla \frac{\partial \phi}{\partial x_{k}}\right|^{2} p \mathrm{~d} x \\
& +2 s \int \frac{\partial \phi}{\partial x_{k}} \beta(s x)(\nabla \beta)(s x) \cdot\left(\nabla \frac{\partial \phi}{\partial x_{k}}\right) p \mathrm{~d} x .
\end{aligned}
$$

Clearly the second term is bounded by

$$
\begin{aligned}
& 2 s\|\nabla \beta\|_{L^{\infty}\left(\mathbb{R}^{d}\right)} \int\left|\frac{\partial \phi}{\partial x_{k}}\right| \beta(s x)\left|\nabla \frac{\partial \phi}{\partial x_{k}}\right| p \mathrm{~d} x \\
& \quad \leq s\|\nabla \beta\|_{L^{\infty}\left(\mathbb{R}^{d}\right)} \int\left[\left(\frac{\partial \phi}{\partial x_{k}}\right)^{2}+\beta(s x)^{2}\left|\nabla \frac{\partial \phi}{\partial x_{k}}\right|^{2}\right] p \mathrm{~d} x
\end{aligned}
$$

and so

$$
\begin{array}{r}
\left(1-s\|\nabla \beta\|_{L^{\infty}\left(\mathbb{R}^{d}\right)}\right) \int \beta(s x)^{2}\left|\nabla \frac{\partial \phi}{\partial x_{k}}\right|^{2} p \mathrm{~d} x \\
-s\|\nabla \beta\|_{L^{\infty}\left(\mathbb{R}^{d}\right)} \int\left(\frac{\partial \phi}{\partial x_{k}}\right)^{2} \mathrm{~d} x \leq \text { LHS. }
\end{array}
$$

Letting $s \rightarrow 0$ in both the LHS and RHS of (A.9), and recalling that $\beta(x)$ is radially decreasing, we conclude from the monotone convergence theorem that

$$
\int\left|\nabla \frac{\partial \phi}{\partial x_{k}}\right|^{2} p \mathrm{~d} x \leq \int \frac{\partial \phi}{\partial x_{k}} G_{k} p \mathrm{~d} x .
$$

Summing over $k$ completes the proof of the lemma.

Next we prove (14). First,

$$
\int|\nabla \phi|^{2} p \mathrm{~d} x \leq \lambda^{-1} \int|h-\hat{h}|^{2} p \mathrm{~d} x \leq \lambda^{-2} \int|\nabla h|^{2} p \mathrm{~d} x
$$

by (13) followed by (A.5) applied to the function $h-$ $\hat{h} \in H_{0}^{1}\left(\mathbb{R}^{d} ; p\right)$. Second, by the definition of $G$, the $L^{2}$ triangle inequality, and (A.10), we show that

$$
\begin{aligned}
& \left(\int|G|^{2} p \mathrm{~d} x\right)^{1 / 2} \\
& \leq\left\|D^{2}(\log p)\right\|_{\infty}\left(\int|\nabla \phi|^{2} p \mathrm{~d} x\right)^{1 / 2}+\left(\int|\nabla h|^{2} p \mathrm{~d} x\right)^{1 / 2} \\
& \leq\left(\frac{\left\|D^{2}(\log p)\right\|_{\infty}}{\lambda}+1\right)\left(\int|\nabla h|^{2} p \mathrm{~d} x\right)^{1 / 2} .
\end{aligned}
$$

Now we take (A.6) and apply Cauchy-Schwarz, followed by (A.10) and (A.12), to find:

$$
\begin{aligned}
& \int\left|D^{2} \phi\right|^{2} p \mathrm{~d} x \\
& \leq\left(\int|\nabla \phi|^{2} p \mathrm{~d} x\right)^{1 / 2}\left(\int|G|^{2} p \mathrm{~d} x\right)^{1 / 2} \\
& \leq\left(\lambda^{-2} \int|\nabla h|^{2} p \mathrm{~d} x\right)^{1 / 2} \\
& \quad\left(\frac{\left\|D^{2}(\log p)\right\|_{\infty}}{\lambda}+1\right)\left(\int|\nabla h|^{2} p \mathrm{~d} x\right)^{1 / 2} \\
& =\lambda^{-2}\left(\lambda+\left\|D^{2}(\log p)\right\|_{\infty}\right) \int|\nabla h|^{2} p \mathrm{~d} x,
\end{aligned}
$$

which proves (14). 


\section{A.3 Proof of Cor. 1}

The first bound follows from (13):

$\mathrm{E}\left[\sum_{l, j}\left|\mathrm{~K}_{l j}\right|^{2}\right]=\sum_{j=1}^{m} \mathrm{E}\left[\left|\nabla \phi_{j}\right|^{2}\right] \leq \sum_{j=1}^{m} \frac{1}{\lambda} \int\left|h_{j}-\hat{h}_{j}\right|^{2} p(x, t) \mathrm{d} x$.

To obtain the second bound, first recall (see (10)),

$$
u_{l}=-\frac{1}{2} \sum_{j=1}^{m} K_{l j}\left(h_{j}+\hat{h}_{j}\right)+\frac{1}{2} \sum_{k=1}^{d} \sum_{j=1}^{m} \mathrm{~K}_{k j} \frac{\partial \mathrm{K}_{l j}}{\partial X_{k}} .
$$

Therefore, using (14),

$$
\begin{aligned}
& \mathrm{E}\left[\sum_{l=1}^{d}\left|u_{l}\right|\right] \\
& \leq \sum_{l, j} \int\left|\mathrm{K}_{l j}\left(h_{j}+\hat{h}_{j}\right)\right| p(x) \mathrm{d} x+\sum_{l, k, j} \int\left|\mathrm{K}_{k j} \frac{\partial^{2} \phi_{j}}{\partial x_{l} \partial x_{k}}\right| p(x) \mathrm{d} x \\
& \leq \text { (const.) } \sum_{j=1}^{m}\left[\int\left|h_{j}\right|^{2} p(x) \mathrm{d} x+\int\left|\nabla h_{j}\right|^{2} p(x) \mathrm{d} x\right] .
\end{aligned}
$$

\section{References}

[1] M. S. Arulampalam, S. Maskell, and N. Gordon. A tutorial on particle filters for online nonlinear/non-Gaussian Bayesian tracking. IEEE Trans. Signal Process., 50:174-188, 2002.

[2] A. Bain and D. Crisan. Fundamentals of Stochastic Filtering. Springer, Cambridge, Mass, 2010.

[3] Y. Bar-Shalom, T. Kirubarajan, and X.-R. Li. Estimation with Applications to Tracking and Navigation. John Wiley \& Sons, Inc., New York, NY, USA, 2002.

[4] T. Bengtsson, P. Bickel, and B. Li. Curse-of-dimensionality revisited: Collapse of the particle filter in very large scale systems. In D. Nolan and T. Speed, editors, Probability and Statistics: Essays in Honor of David A. Freedman, volume 2, pages 316-334. Institute of Mathematical Statistics, 2008.

[5] K. Bergemann and S. Reich. An ensemble Kalman-Bucy filter for continuous data assimilation. Meteorologische Zeitschrift, 21(3):213219, 062012.

[6] K. Berntorp. Feedback particle filter: Application and evaluation. In Proc. $18^{\text {th }}$ Int. Conf. on Inf. Fusion, pages 1633-1640, July 2015.

[7] A. Budhiraja, L. Chen, and C. Lee. A survey of numerical methods for nonlinear filtering problems. Physica D: Nonlinear Phenomena, 230(1-2):27-36, 2007.

[8] D. Crisan and J. Xiong. Approximate McKean-Vlasov representations for a class of SPDEs. Stochastics: An International Journal of Probability and Stochastic Processes, pages 1-16, 2009.

[9] F. Daum and J. Huang. Generalized particle flow for nonlinear filters. In Proc. SPIE, volume 7698, April 2010.

[10] F. Daum and J. Huang. Particle degeneracy: root cause and solution. In Proc. SPIE, volume 8050, May 2011.

[11] P. Del Moral. Mean field simulation for Monte Carlo integration. Monographs on Statistics \& Applied Probability. Chapman \& Hall/CRC, June 2013.

[12] P. Del Moral, F. Patras, and S. Rubenthaler. A mean field theory of nonlinear filtering. In D. Crisan and B. L. Rozovskii, editors, The Oxford Handbook of Nonlinear Filtering, Oxford Handbooks in Mathematics, pages 705-740. Oxford University Press, 2011.
[13] P. Del Moral and E. Rio. Concentration inequalities for mean field particle models. Ann. Appl. Probab., 21(3):1017-1052, 2011.

[14] A. Doucet, N. de Freitas, and N. Gordon. Sequential Monte-Carlo Methods in Practice. Springer-Verlag, April 2001.

[15] A. Doucet, S. Godsill, and C. Andrieu. On sequential Monte Carlo sampling methods for Bayesian filtering. Statistics and computing, 10(3):197-208, 2000.

[16] A. Doucet and A. M. Johansen. A tutorial on particle filtering and smoothing: fifteen years later. In Oxford Handbook of Nonlinear Filtering, pages 656-704, 2011.

[17] L. C. Evans. Partial Differential Equations. American Mathematical Society, Providence, RI, 1998.

[18] G. Evensen. Sequential data assimilation with a nonlinear quasigeostrophic model using Monte Carlo methods to forecast error statistics. J. Geophys. Res, 99:10143-10162, 1994.

[19] M. A. Fallah, R. P. Malhamé, and F. Martinelli. Decentralized estimation in a class of measurements induced mean field control problems. In Proc. 52 ${ }^{\text {nd }}$ IEEE Conf. Decision Contr., pages 31463151, Florence, Italy, December 2013.

[20] M. A. Fallah, R. P Malhamé, and F. Martinelli. Distributed estimation and control for large population stochastic multi-agent systems with coupling in the measurements. In Proc. European Control Conf., pages 4353-4358, Zürich, Switzerland, July 2013.

[21] W. R. Gilks and C. Berzuini. Following a moving target - Monte Carlo inference for dynamic Bayesian models. J. R. Statist. Soc. B, 63(1):127-146, 2001.

[22] P. W. Glynn and S. P. Meyn. A Liapounov bound for solutions of the Poisson equation. Ann. Appl. Probab., 24(2):916-931, 1996.

[23] N. J. Gordon, D. J. Salmond, and A. F. M. Smith. Novel approach to nonlinear/non-Gaussian Bayesian state estimation. IEE Proc. F Radar and Signal Processing, 140(2):107-113, 1993.

[24] B. Helffer and F. Nier. Criteria to the Poincaré inequality associated with Dirichlet forms in $\mathbb{R}^{d}, d \geq 2$. Int. Math. Res. Not., (22):11991223, 2003.

[25] M. Huang, P. E. Caines, and R. P. Malhame. Large-population costcoupled LQG problems with nonuniform agents: Individual-mass behavior and decentralized $\varepsilon$-Nash equilibria. IEEE Trans. Autom. Control, 52(9):1560-1571, 2007.

[26] J. Johnsen. On the spectral properties of Witten-Laplacians, their range projections and Brascamp-Lieb's inequality. Integral Equations Operator Theory, 36(3):288-324, 2000.

[27] B. Jourdain and F. Malrieu. Propagation of chaos and Poincaré inequalities for a system of particles interacting through their CDF. Ann. Appl. Probab., 18(5):1706-1736, 102008.

[28] G. Kitgawa. Monte Carlo filter and smoother for non-Gaussian nonlinear state space models. J. Comput. Graph. Statist., 5(1):1-25, 1996.

[29] P. Kloeden and E. Platen. Numerical solution of stochastic differential equations. Springer-Verlag, 1992.

[30] H. J. Kushner. On the differential equations satisfied by conditional probablitity densities of Markov processes, with applications. $J$. SIAM Control Ser. A, 2(1):106-119, 1964.

[31] J. M. Lasry and P. L. Lions. Mean field games. Japan. J. Math., 2:229-260, 2007.

[32] R. S. Laugesen, P. G. Mehta, S. P. Meyn, and M. Raginsky. Poisson's equation in nonlinear filtering. SIAM J. Control and Optimization, 53(1):501-525, 2015.

[33] F. Le Gland, V. Monbet, and V.-D. Tran. Large sample asymptotics for the ensemble Kalman filter. Research Report RR-7014, 2009.

[34] R. Ma and T. P. Coleman. Generalizing the posterior matching scheme to higher dimensions via optimal transportation. In Allerton 
Conf. on Communication, Control and Computing, pages 96 - 102, 2011.

[35] S. Méléard. Asymptotic behaviour of some interacting particle systems; McKean-Vlasov and Boltzmann models. In D. Talay and L. Tubaro, editors, Probabilistic Models for Nonlinear Partial Differential Equations, volume 1627 of Lecture Notes in Mathematics, pages 42-95. Springer Berlin Heidelberg, 1996.

[36] S. K. Mitter and N. J. Newton. A variational approach to nonlinear estimation. SIAM J. Control and Optimization, 42(5):1813-1833, 2003.

[37] P. Del Moral. Feynman-Kac Formulae: Genealogical and Interacting Particle Systems with Applications. Springer, New York, 2004.

[38] C. Musso, N. Oudjane, and F. Gland. Improving regularised particle filters. In Sequential Monte Carlo Methods in Practice, Statistics for Engineering and Information Science, pages 247-271. Springer New York, 2001.

[39] B. K. Øksendal. Stochastic Differential Equations: An Introduction with Applications. Springer, Berlin, 2003.

[40] S. Pequito, A. Aguiar, B. Sinopoli, and D. A. Gomes. Nonlinear estimation using mean field games. In Proc. $5^{\text {th }}$ International Conf. Network Games, Control and Optimization, pages 1-5. IEEE, 2011.

[41] M. K. Pitt and N. Shephard. Filtering via simulation: auxiliary particle filters. J. Amer. Statist. Assoc., 94(446):590-599, 1999.

[42] S. Reich. A dynamical systems framework for intermittent data assimilation. BIT Numerical Mathematics, 51(1):235-249, 2011.

[43] T. Schon, F. Gustafsson, and P. J. Nordlund. Marginalized particle filters for mixed linear/nonlinear state-space models. IEEE Trans. Signal Process., 53(7):2279-2289, July 2005.

[44] P. M. Stano. Nonlinear State and Parameter Estimation for Hopper Dredgers. dissertation, Delft University of Technology, 2013.

[45] P. M. Stano, A. K Tilton, and R. Babuska. Estimation of the soil-dependent time-varying parameters of the hopper sedimentation model: The FPF versus the BPF. Control Engineering Practice, 24:67-78, 2014

[46] R. L. Stratonovich. Conditional Markov processes. Theory of Probability \& Its Applications, 5(2):156-178, 1960.

[47] A. S. Sznitman. Topics in propagation of chaos. In P.-L. Hennequin, editor, Ecole d'Eté de Probabilités de Saint-Flour XIX 1989, volume 1464 of Lecture Notes in Mathematics, pages 165-251. Springer Berlin Heidelberg, 1991.

[48] A. K. Tilton, S. Ghiotto, and P. G. Mehta. A comparative study of nonlinar filtering techniques. In Proc. $16^{\text {th }}$ Int. Conf. on Inf. Fusion, pages 1827-1834, July 2013.

[49] A. K. Tilton, E. T. Hsiao-Wecksler, and P. G. Mehta. Filtering with rhythms: Application of the feedback particle filter to estimation of human gait cycle. In Proc. Amer. Control Conf., pages 3433-3438, Montréal, Canada, June 2012.

[50] A. K. Tilton, P. G. Mehta, and S. P. Meyn. Multi-dimensional feedback particle filter for coupled oscillators. In Proc. Amer. Control Conf., pages 2415-2421, Washington D.C., June 2013.

[51] J. Xiong. Particle approximations to the filtering problem in continuous time. In The Oxford handbook of nonlinear filtering, pages 635-655. Oxford Univ. Press, Oxford, 2011.

[52] T. Yang, H. A. P. Blom, and P. G. Mehta. The continuous-discrete time feedback particle filter. In Proc. Amer. Control Conf., pages 648-653, Portland, OR, June 2014.

[53] T. Yang, R. S. Laugesen, P. G. Mehta, and S. P. Meyn. Multivariable feedback particle filter. In Proc. $51^{\text {st }}$ IEEE Conf. Decision Contr. pages 4063-4070, Maui, HI, December 2012.

[54] T. Yang, P. G. Mehta, and S. P. Meyn. Feedback particle filter with mean-field coupling. In Proc. $50^{\text {th }}$ IEEE Conf. Decision Contr. pages 7909-7916, Orlando, FL, December 2011.
[55] T. Yang, P. G. Mehta, and S. P. Meyn. A mean-field control-oriented approach for particle filtering. In Proc. Amer. Control Conf., pages 2037-2043, San Francisco, June 2011.

[56] T. Yang, P. G. Mehta, and S. P. Meyn. Feedback particle filter. IEEE Trans. Autom. Control, 58(10):2465-2480, October 2013.

[57] H. Yin, P. G. Mehta, S. P. Meyn, and U. V. Shanbhag. Synchronization of coupled oscillators is a game. In Proc. Amer. Control Conf., pages 1783-1790, Baltimore, June 2010. 